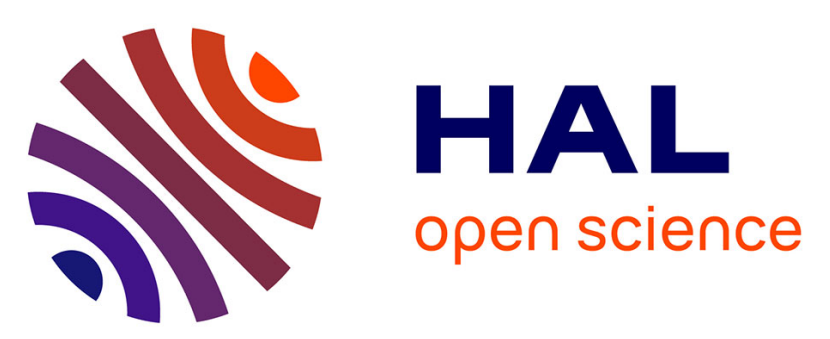

\title{
The effects of terrestrial weathering on samarium-neodymium isotopic composition of ordinary chondrites
}

Hamed Pourkhorsandi, Vinciane Debaille, Rosalind M.G. Armytage, Matthias van Ginneken, Pierre Rochette, J. Gattacceca

\section{To cite this version:}

Hamed Pourkhorsandi, Vinciane Debaille, Rosalind M.G. Armytage, Matthias van Ginneken, Pierre Rochette, et al.. The effects of terrestrial weathering on samarium-neodymium isotopic composition of ordinary chondrites. Chemical Geology, 2021, pp.120056. 10.1016/j.chemgeo.2020.120056 . hal03098323

\section{HAL Id: hal-03098323 \\ https://hal.science/hal-03098323}

Submitted on 13 Jan 2021

HAL is a multi-disciplinary open access archive for the deposit and dissemination of scientific research documents, whether they are published or not. The documents may come from teaching and research institutions in France or abroad, or from public or private research centers.
L'archive ouverte pluridisciplinaire HAL, est destinée au dépôt et à la diffusion de documents scientifiques de niveau recherche, publiés ou non, émanant des établissements d'enseignement et de recherche français ou étrangers, des laboratoires publics ou privés. 


\section{Journal Pre-proof}

The effects of terrestrial weathering on samarium-neodymium isotopic composition of ordinary chondrites

Hamed Pourkhorsandi, Vinciane Debaille, Rosalind M.G. Armytage, Matthias van Ginneken, Pierre Rochette, Jérôme Gattacceca

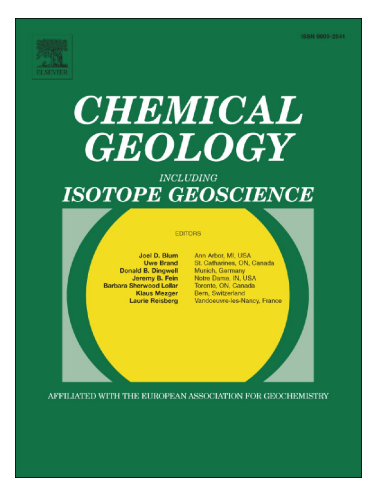

PII: $\quad$ S0009-2541(20)30595-7

DOI: $\quad$ https://doi.org/10.1016/j.chemgeo.2020.120056

Reference: $\quad$ CHEMGE 120056

To appear in: $\quad$ Chemical Geology

Received date: $\quad 24$ February 2020

Revised date: $\quad 4$ May 2020

Accepted date: $\quad 30$ December 2020

Please cite this article as: H. Pourkhorsandi, V. Debaille, R.M.G. Armytage, et al., The effects of terrestrial weathering on samarium-neodymium isotopic composition of ordinary chondrites, Chemical Geology (2021), https://doi.org/10.1016/ j.chemgeo.2020.120056

This is a PDF file of an article that has undergone enhancements after acceptance, such as the addition of a cover page and metadata, and formatting for readability, but it is not yet the definitive version of record. This version will undergo additional copyediting, typesetting and review before it is published in its final form, but we are providing this version to give early visibility of the article. Please note that, during the production process, errors may be discovered which could affect the content, and all legal disclaimers that apply to the journal pertain.

(C) 2021 Published by Elsevier. 


\section{The effects of terrestrial weathering on samarium-neodymium isotopic composition of ordinary chondrites}

Hamed Pourkhorsandi ${ }^{a, b, 1^{\star}}$ hamed.pourkhorsandi@ulb.ac.be, Vinciane Debaille ${ }^{a}$, Rosalind M. G. Armytage ${ }^{\mathrm{a}, \mathrm{c}}$, Matthias van Ginneken ${ }^{\mathrm{a}, \mathrm{d}}$, Pierre Rochette ${ }^{\mathrm{b}}$, Jérôme Gattaccecab $^{\text {b }}$

`Laboratoire G-Time, Université Libre de Bruxelles, CP 160/02, 50, Av. F.D. Roosevelt, 1050 Brussels, Belgium

${ }^{\mathrm{b}}$ Aix-Marseille Univ, CNRS, IRD, Coll. France, INRAE, CE EGE, Aix-en-Provence, France

`Jacobs/JETS, NASA Johnson Space Center, 2101 Ni.CA Parkway, Mailcode XI3, Houston, TX, 77058, USA

${ }^{\text {d} R o y a l ~ B e l g i u m ~ I n s t i t u t e ~ o f ~ N a t u r a l ~ S c i e n c e s ~:-' e ~ V a u t i e r ~ 29, ~ B-1000 ~ B r u x e l l e s, ~}$ Belgium

"Corresponding author.

\section{Abstract:}

Following their fall to Earth, , neteorites experience weathering. In this systematic study, we evaluate the trace $9.16 m e n t$ composition of ordinary chondrites from the Antarctic cold desert, a A Aiacama (Chile) and Lut (Iran) hot deserts, with an emphasis on rare eat h viements (REE). Our data confirms that terrestrial weathering of meteorites in hot duverts changes their trace element ( $\mathrm{Sr}, \mathrm{Ba}, \mathrm{REE}, \mathrm{Hf}$, Th, and U) concentrations. However, weathering effects in majority of Antarctic samples are limited to slight $\mathrm{Ba}, \mathrm{REE}, \mathrm{Hf}$, and Th depletions and in some case to $\mathrm{U}$ enrichment. In comparison to the Antarctic meteorites, hot desert samples show greater disturbances and REE fractionation relative to the average fall values. We measured the Sm-Nd isotopic composition of the hot desert meteorites that have heavily

\footnotetext{
${ }^{1}$ Present address: Laboratoire G-Time, Université Libre de Bruxelles, CP 160/02, 50, Av. F.D. Roosevelt, 1050 Brussels, Belgium
} 
affected REE compositions. Our Sm-Nd isotopic data show a significant effect of terrestrial weathering evidenced by non-CHUR ${ }^{147} \mathrm{Sm} /{ }^{144} \mathrm{Nd}$ and ${ }^{143} \mathrm{Nd} /{ }^{144} \mathrm{Nd}$ ratios. Measurements show a higher variation and lower values of ${ }^{147} \mathrm{Sm} /{ }^{144} \mathrm{Nd}$ for the Atacama samples than those from the Lut Desert. Deviations from CHUR ${ }^{147} \mathrm{Sm} /{ }^{144} \mathrm{Nd}$ value are in positive accordance with the degree of La/Lu fractionation caused by weathering. The $\varepsilon N d$ values of Atacama and Lut deserts meteorites range from -2.20 to +1.61 , which is wider than the -1.07 to +0.64 range for falls. We suggest that disturbance of primary $\mathrm{Sm} / \mathrm{Nd}$ ratios resulting from mixing with terrestrial components originating from soil during weathering is responsible for lower ${ }^{147} \mathrm{Sm} /{ }^{144} \mathrm{Nd}$ ratio in these meteorites. The majority of the $/ \mathrm{i}^{7} \mathrm{c}$ cama meteorites regardless of their weathering degrees have their REE ( $\mathrm{mm}$ ) ositions and $\varepsilon \mathrm{Nd}$ affected by terrestrial contamination. Both ${ }^{147} \mathrm{Sm} /{ }^{144} \mathrm{~N} d$ ra io and $\varepsilon N d$ values show no straightforward relationship with weathering degree. : 'towever, in both cases the samples with the highest negative isotopic distur an jes are $\mathrm{H}$ chondrites from the Atacama and Lut deserts. In addition, $\mathrm{Ba}$ co $\backsim$ intration shows a negative correlation with ${ }^{147} \mathrm{Sm} /{ }^{144} \mathrm{Nd}$ ratio. Care must be take: in $\mathrm{u}$ account while dealing with samples collected from hot deserts, even fres' -lc sking ones.

Keywords: Sm-Nd, Samarium Neodyrnium, Chondrite, Weathering, Hot desert, Contamination

\section{INTRODUCTION}

The systematics of rars ea th elements (REE) and the Sm-Nd isotopic chronometer are esse tial in petrogenetic and radiometric studies of terrestrial and extraterrestrial rocks (૯. .., DePaolo, 1988). As a result of their slightly different nuclear and chemical properties, REE respond to common petrological processes, such as partial melting and partial evaporation, by developing fractionated light-REE (from La to Sm) or heavy-REE (from Eu to Lu) elemental patterns (e.g., Hanson, 1980; Davis and Richter, 2014). Radioactive decay of ${ }^{147} \mathrm{Sm}\left(\mathrm{t}_{1 / 2}=106\right.$ Gyr) to ${ }^{143} \mathrm{Nd}$ is an important tracer for chemical differentiation processes affecting the REE during planetary evolution. In addition, the $\mathrm{Sm}-\mathrm{Nd}$ isotopic system is one of the most precise and useful dating methods in geology. These properties make REE and Sm-Nd isotopes powerful tools to study the petrogenesis and origin of different magmatic rocks. 
Except for returned samples from a few objects such as the Moon and one asteroid 25143 Itokawa and comet Wild 2, excluding small objects such a interplanetary dust particles, meteorites are the only samples available from other solar system bodies. Meteorites allow studying the formation, evolution, and structure of the solar system. Ongoing developments in analytical chemistry, improved elemental and isotopic measurements, and accessibility of more meteoritic material has led us to have a comprehensive though still incomplete vision about the early stages of solar system evolution. These data enable us to see relationships between different meteorite groups, their formation ages, petrogenesis, etc.

The vast majority ( 98\%) of meteorites available for stu'i,' are collected in hot and cold deserts. This is particularly the case for some rare net orite types such as Martian and Lunar meteorites, angrites, etc. These $\mathrm{m}$. „tec rites collected in deserts are referred to as finds, and unlike observed falls. $h_{a}$ 'e had relatively long residence times on Earth. Their terrestrial ages range from ، ’n, of thousands years (kyr) for most hot deserts finds (Jull et al., 2013) to h's1, yreds of kyr for Antarctica and Atacama finds (Drouard et al., 2019; Welt $n e_{\iota}$ al., 1997). The exposure of meteorites to terrestrial environments during thic re', idence time alters their mineralogy, chemistry, and isotopic properties '๑.g., Bland et al., 2006). Meteorite weathering is a complex process controlled by dif $i e$ in factors including terrestrial residence time, climate, soil composition at the re cuvery site, meteorite type, size, and shape (e.g., Pourkhorsandi et al., 2017a, Hotınann et al., 2018). As observed in samples collected from different regions of the - iacama desert, meteorite weathering can be variable at sub-regional scale (ML nay :o et al., 2013). Understanding meteorite weathering processes is critical to c void any data misinterpretation while working on meteorite finds. Terrestrial weathering of extraterrestrial materials is not only limited to "old" finds, but can occur during the short time span between meteorite falls and their recovery (Bischoff et al., 2011; Walker et al., 2018), and even during laboratory storage of samples returned by space missions (Velbel, 2014). All of this evidence points to a potential "threat" from terrestrial weathering on the integrity of cosmochemical data obtained from finds and show the importance of a detailed documentation of meteorite weathering processes.

Most of the studies of meteorite weathering have dealt with mineralogy and major element geochemistry (Bland et al., 2006; Golden et al., 1995; Gooding, 1982; King et al., 2020; Velbel et al., 1991). A few studies also have focused on particular trace 
elements such as Ba, Sr, and REE (Al-Kathiri et al., 2005; Crozaz et al., 2003; Pourkhorsandi et al., 2017a; Shimizu et al., 1983; Stelzner et al., 1999; Zurfluh et al., 2011). The number of such studies is smaller when it comes to isotopic investigations. However, paucity of information on this topic does not correlate with its importance. In their work on the observed CM chondrite fall Sutter's Mill, Walker et al. (2018) showed disturbances in ${ }^{187} \mathrm{Re}^{-{ }^{187}} \mathrm{Os}$ and $\mathrm{Re} / \mathrm{Os}$ ratios in the fragments which had experienced only one post-fall rain event. Observing these effects on a fall meteorite emphasizes the importance of this matter. Isotopic changes caused by meteorite weathering are also reported in $\mathrm{H}$ (Stephant et al., 2018), O (Stelzner and Heide, 1996), noble gases (Cartwright et al., 2010; Kaneo's 1983; Schultz et al., 2005), Sr (Borg et al., 2016, 2003; Brandon et al., 2004; Ela do et al., 2014; Shih et al., 2007), Hf (Sokol et al., 2008; Tatsumoto et al., 19 31), Os (Borg et al., 2003; Brandon et al., 2012), Fe (Saunier et al., 2010), U- a.'d Th- series as well as Cs (Weber et al., 2017), and $\mathrm{Pb}$ (Tatsumoto et al., 1281, isotopes.

The terrestrial weathering effects on metf vi 'tes' Sm-Nd isotopic system, the focus of this work, are contradictory. For examp' ?, (urandon et al., 2004; Debaille et al., 2007; Elardo et al., 2014; Haloda et ¿'. ' .009) report effects of hot desert weathering in their measurements on Martian meteorites to be insignificant. Meanwhile, most of the studies such as (Borg et al., iv ₹. Edmunson et al., 2005; Shih et al., 2007) and most of the studies on desert $r$ i $i$ : urites do not report any Sm-Nd isotopic disturbances in the weather $\gamma$ meteorites with already disturbed Rb-Sr systematics.

Despite their importance ir. cosmochemical studies, REE and especially the Sm-

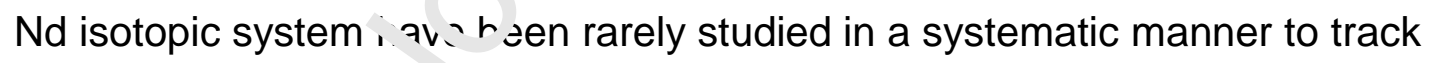
meteorite weathering of ects on their composition. In this study we evaluate REE composition of ordinary chondrites from Antarctica, Atacama and Lut hot deserts. We also analyzed the Sm-Nd isotopic systematics of meteorites with heavily affected REE compositions.

Ordinary chondrites are the most abundant meteorite types among falls and finds. Meteorites with different weathering degrees, as determined by their mineralogy, were analyzed in order to pinpoint the relationships between REE composition in meteorites with the region where they were found and weathering degree. The second goal of this work is to investigate the effects of weathering on Sm-Nd isotopic composition of meteorites from hot deserts. 


\section{SAMPLES AND ANALYSES}

\subsection{Studied meteorites}

Table 1 shows the list of the ordinary chondrites used in this study. Hot desert and Antarctic samples are from the CEREGE (Aix-en-Provence, France) and Royal Belgium Institute of Natural Sciences (Brussels, Belgium) collections, respectively. Ferromagnesian silicates (olivine and pyroxene), ( $\mathrm{Fe}, \mathrm{Ni})$ metal, and troilite ( $\mathrm{FeS}$ ) are the main primary components of this type of meteorites (Weisberg et al., 2006). During terrestrial weathering they alter to Fe oxyhydroxides (e.g., Pourkhorsandi et al., 2019). To study the possible effects of the meteorite find, ?gion on the trace elements and $\mathrm{Sm}-\mathrm{Nd}$ isotopic composition, meteorites fru $m$ ine Asuka dense collection area (DCA) in Antarctica (Zekollari et al., ? $\iota^{1} a^{\prime}$ ), the Atacama (Chile) (Drouard et al., 2019; Hutzler et al., 2016; Gattac cec $z$ et al., 2011) and Lut (Iran) (Pourkhorsandi et al., 2019) hot deserts were se'eci?d. In order to observe possible effects of $(\mathrm{Fe}, \mathrm{Ni})$ metal abundance and min (ral ; weathering, we chose members from ' ' 'h, ' and L groups, as well as LL for Antarctic samples. These three are the main gru. ns of ordinary chondrites, which are distinguished by having different $(\mathrm{F} \in, \mathrm{Ni}$ ) metal and total $\mathrm{Fe}$ contents. $\mathrm{H}$ chondrites have higher ( $\mathrm{Fe}, \mathrm{Ni}$ ) metal and tota' $\mathrm{F}=$ than members of $\mathrm{L}$ (low total $\mathrm{Fe}$ ) and $\mathrm{LL}$ groups (low metal and total Fc corrients) (Wasson and Kallemeyn, 1988). To avoid analyzing possible isotopic? $\|_{y}$ ' Inequilibrated exotic grains, most of the analyzed meteorites were selected 1,$\urcorner r, 1$ thermally equilibrated petrologic types (4 to 6 ) . Finally, meteorites $v^{\text {ith }}$ vifferent weathering degrees were selected. Weathering scales differ between $\Delta$ itarctic and other meteorites. For the Antarctic meteorites, the Meteorite Working Group at NASA and NIPR use weathering categories "A," "B," and "C" to denote minor, moderate, and severe rustiness at a macroscopic scale. For hot desert weathering, the scale is based on microscopic observations: W2 denotes moderate oxidation of metal (20-60\% being affected), W3 for heavy oxidation of metal and troilite (60-95\% being replaced), and W4 for complete (>95\%) oxidation of metal and troilite (Wlotzka, 1993).

Table 1. Studied meteorites listed based on their find locations.

\begin{tabular}{|l|c|c|}
\hline Meteorite & Type & Weathering degree \\
\hline
\end{tabular}




\begin{tabular}{|c|c|c|}
\hline \multicolumn{3}{|l|}{ Atacama Desert } \\
\hline Caleta el Cobre $006^{1}$ & L6 & W3 \\
\hline El Médano $023^{1}$ & L6 & W3 \\
\hline El Médano $049^{2}$ & $\mathrm{H} 4$ & W3 \\
\hline El Médano $089^{3}$ & L6 & W4 \\
\hline El Médano $090^{3}$ & L6 & W2 \\
\hline El Médano $091^{3}$ & $\mathrm{H} 5$ & W3 \\
\hline El Médano $111^{3}$ & $\mathrm{H} 5$ & W4 \\
\hline El Médano $120^{3}$ & $\mathrm{H} 5$ & W2 \\
\hline \multicolumn{3}{|l|}{ Lut Desert } \\
\hline Kerman $002^{4}$ & L6 & W3 \\
\hline Kerman $003^{5}$ & L5 & $\overline{W 2}=-$ \\
\hline Kerman $009^{6}$ & $\mathrm{H} 5$ & v.? \\
\hline Kerman $013^{6}$ & $\mathrm{H} 5$ & $\sqrt{13}$ \\
\hline Kerman $026^{6}$ & L6 & $\overline{\mathrm{N} 2}$ \\
\hline Shahdad $^{7}$ & H5 & W4 \\
\hline \multicolumn{3}{|l|}{ Antarctica } \\
\hline Asuka $09135^{4}$ & $L^{\prime} .3$ & B \\
\hline Asuka $09387^{4}$ & $\mathrm{H}_{4}$ & $\mathrm{~B} / \mathrm{C}$ \\
\hline Asuka $09436^{4}$ & :-13 & $\mathrm{C}$ \\
\hline Asuka $09455^{4}$ & L6 & $\mathrm{B} / \mathrm{C}$ \\
\hline Asuka $09516^{4}$ & $\mathrm{H} 6$ & $\mathrm{C}$ \\
\hline Asuka $09618^{4}$ & H5 & $\mathrm{C}$ \\
\hline Asuka $10091^{4}$ & LL6 & B \\
\hline Asuka $10182^{4}$ & $\mathrm{H} 5$ & $\mathrm{~A} / \mathrm{B}$ \\
\hline Asuka $10187^{4}$ & L5 & B \\
\hline Asuka $10224^{4}$ & L4 & $A$ \\
\hline Asuka $12056^{4}$ & L4 & $A$ \\
\hline
\end{tabular}

${ }^{1}$ (Ruzicka et al., 2014); ${ }^{2}$ (Ruzicka et al., 2015a) ; ${ }^{3}$ (Ruzicka et al., 2015b) ; ${ }^{4}$ (Ruzicka et al., 2017) ; ${ }^{5}$ (Bouvier et al., 2017a) ; ${ }^{6}$ (Bouvier et al., 2017b) ; ${ }^{7}$ (Garvie, 2012) ;

\subsection{Analytical work}

Using an optical petrographic microscope, meteorite fragments without visible attached or imbedded terrestrial grains, cracks, and desert varnish were chosen. Between 500 to $1000 \mathrm{mg}$ of the fragments were crushed using an agate pestle and mortar, specifically used for chondritic samples. 
All the elemental and isotopic analyses were carried out at the Laboratoire G-Time of the Université Libre de Bruxelles, Belgium. The analytical methodology used in this study is similar to that used by (Armytage et al., 2018; Wainwright et al., 2019).

For trace element analyses approximately $200 \mathrm{mg}$ of powdered samples were dissolved using concentrated acid $\left(\mathrm{HF}+\mathrm{HNO}_{3}\right.$ followed by $\left.\mathrm{HCl}\right)$ in sealed Savillex beakers on a hot plate at $120^{\circ} \mathrm{C}$ for four days with evaporation and re-dissolution between the two acid steps. Elementals analyses were conducted using an Agilent 7700 Quadrupole-Inductively Coupled Plasma-Mass Spectrometer (Q-ICP-MS) using a He collision cell. Samples were introduced with $5 \% \mathrm{HN}_{3}$, and In was used as an internal standard. Standards BHVO-2 and BCR-2 were unsa :s international reference material. Table 2 shows a comparison of the these standards obtained during this work with thos $\geq 1, n r .1$ the literature.

Table 2: Whole-rock concentrations of trace e' ement (in $\mu \mathrm{g} / \mathrm{g}$ ) in the analyzed standards compared to the literature valı,

\begin{tabular}{|c|c|c|c|c|}
\hline $\begin{array}{l}\text { Elem } \\
\text { ent }\end{array}$ & $\begin{array}{l}\text { BHVO-2 (this } \\
\text { work) }\end{array}$ & $\begin{array}{c}\text { BHVi ? } \\
\text { (lit, vrature) }\end{array}$ & $\begin{array}{r}\text { BCR-2 (this } \\
\text { work) }\end{array}$ & $\begin{array}{c}\text { BCR-2 } \\
\text { (literature) }\end{array}$ \\
\hline $\mathrm{Sr}$ & 399 & $-29-23$ & 292.2 & $346 \pm 14$ \\
\hline $\mathrm{Nb}$ & 17 & $18=2$ & 12.1 & $12.4 \pm 0.2$ \\
\hline $\mathrm{Ba}$ & 138 & $30 \pm 13$ & 737.8 & $683 \pm 28$ \\
\hline La & 16 & $15 \pm 1$ & 27.7 & $25 \pm 1$ \\
\hline $\mathrm{Ce}$ & 38 & $38 \pm 2$ & 57.7 & $53 \pm 2$ \\
\hline Pr & 5 & $5.4 \pm 0.03$ & 7.3 & $6.8 \pm 0.3$ \\
\hline Nd & 25 & $25.0 \pm 1.8$ & 29.5 & $28 \pm 2$ \\
\hline $\mathrm{Sm}$ & 6 & $6.2 \pm 0.4$ & 6.8 & $6.7 \pm 0.3$ \\
\hline Eu & $5-$ & $2.0 \pm 0.01$ & 2.2 & $2.0 \pm 0.1$ \\
\hline Gd & 6.4 & $6.3 \pm 0.2$ & 8.2 & $6.8 \pm 0.3$ \\
\hline $\mathrm{Tb}$ & 0.9 & 0.9 & 1.2 & $1.07 \pm 0.04$ \\
\hline Dy & 5 & $5.3 \pm 0.03$ & 6.8 & $6.4 \pm 0.05$ \\
\hline Ho & 1.1 & $1.04 \pm 0.04$ & 1.5 & $1.33 \pm 0.06$ \\
\hline Er & 3 & $2.5 \pm 0.01$ & 4 & $3.7 \pm 0.4$ \\
\hline $\mathrm{Tm}$ & 0.4 & 0.3 & 0.6 & 0.54 \\
\hline $\mathrm{Yb}$ & 2 & $2.0 \pm 0.2$ & 3.7 & $2.5 \pm 0.2$ \\
\hline Lu & 0.3 & $0.28 \pm 0.01$ & 0.5 & $0.51 \pm 0.02$ \\
\hline Hf & 4.6 & $4.1 \pm 0.3$ & 5.1 & $4.8 \pm 0.2$ \\
\hline $\mathbf{P b}$ & 2 & $1.6 \pm 0.04$ & 7.8 & $11 \pm 2$ \\
\hline Th & 2 & $1.2 \pm 0.3$ & 6.5 & $6.2 \pm 0.7$ \\
\hline $\mathbf{U}$ & 0.4 & $0.4 \pm 0.03$ & 1.9 & $1.69 \pm 0.19$ \\
\hline
\end{tabular}

${ }^{1}$ (Wilson, 1997a); ${ }^{2}$ (Jochum et al., 2016); ${ }^{3}$ (Wilson, 1997b) 
Based on our trace element data and generally higher interaction of the hot desert meteorites with their environment compared to meteorites from Antarctica (Koeberl and Cassidy, 1991), only hot desert samples were chosen for Sm-Nd isotopic measurements. About $500 \mathrm{mg}$ of each sample were weighed and digested using a mixture of concentrated $\mathrm{HNO}_{3}-\mathrm{HF}$ followed by $\mathrm{HCl}$ (similar to the method described above). Neodymium and Sm were purified from the matrix using a two-column ionexchange technique as detailed in (Armytage et al., 2018). After digestion a 5\% aliquot was taken for isotope dilution analysis of $\mathrm{Nd}$, and $\mathrm{Sm}$ with a ${ }^{150} \mathrm{Sm}-{ }^{148} \mathrm{Nd}$ mixed spike. For both spiked and unspiked samples, first ${ }^{\dagger}$. $:$ were extracted from the sample using $\sim 2 \mathrm{ml}$ of AG50W-X8 cation resin (2nn_..n'J mesh). Then HDEHP (di-2ethylhexyl-orthophosphoric acid)-coated Teflon pu'ser was used for purifying $\mathrm{Nd}$ from $\mathrm{Sm}$ and $\mathrm{Ce}$, both of which cause isobari $; \mathrm{II}{ }^{+}$eiferences. The unspiked $\mathrm{Nd}$ cuts were measured on the Nu-Plasma II Multipl 2 L ᄀllector - Inductively Coupled Plasma -Mass Spectrometer (MC-ICP-MS) it U .B, in dry plasma with an Aridus II desolvator. For unspiked Nd each ane'y is innsists of 3 blocks of 20 runs each. The repeated measurements of Rennes inc national standard gave an internal reproducibility of $13 \mathrm{ppm}(2 \sigma, \mathrm{n}=20)$. Total external reproducibility based on full duplicates is better than $33 \mathrm{ppm}$. N'er dymium isotopic compositions were corrected by internal normalization to thi value of ${ }^{146} \mathrm{Nd} /{ }^{144} \mathrm{Nd}=0.7219$, and by sample-standard bracketing using the recom. $m \in$ ? ded value of the Rennes standard $\left({ }^{143} \mathrm{Nd} /{ }^{144} \mathrm{Nd}=0.511961: \Omega_{1}, 2\right.$, vel and Blichert-Toft, 2001). Total blank was negligible (8 pg) and reproduc.' 'illi:" ' as better than 99\% as measured over seven replicate analyses. The spiked $\beth^{\prime}$ 'quots were measured on the Nu Plasma I MC-ICP-MS at ULB. For analysis of spiked Sm and Nd the mass fractionation factors for each element were derived by solving iteratively non-linear equations combining the exponential fractionation law and the spike natural mixing equations as in (Debaille et al., 2007).

\section{RESULTS}

\subsection{Trace elements}

Whole-rock trace element composition of the analyzed meteorites are reported in Table 3. The Cl-normalized spider diagrams of Antarctica and hot desert samples 
(Atacama and Lut) along with those of average composition of different groups of ordinary chondrites (falls) are shown in Fig. 1. For EM 091, EM 120, and Kerman 026 average values of two cuts are presented.

Table 3: Whole-rock concentrations of trace element (in $\mu \mathrm{g} / \mathrm{g}$ ) in the studied chondrites from the Antarctica, and Atacama and Lut hot deserts. Average $\mathrm{Cl}$ and ordinary chondrites abundances are from (Barrat et al., 2012) and (Wasson and Kallemeyn, 1988), respectively.

\begin{tabular}{|c|c|c|c|c|c|c|c|c|c|c|c|c|c|c|c|c|c|c|c|c|c|c|c|c|}
\hline $\begin{array}{l}\text { Meteo } \\
\text { rite }\end{array}$ & $\begin{array}{l}s \\
r\end{array}$ & $\begin{array}{l}N \\
b\end{array}$ & $\begin{array}{l}\text { B } \\
\text { a }\end{array}$ & $\begin{array}{l}\mathrm{L} \\
\mathrm{a}\end{array}$ & $\begin{array}{l}\text { C } \\
\text { e }\end{array}$ & $\mathrm{Pr}$ & $\begin{array}{l}N \\
d\end{array}$ & $\begin{array}{l}\mathrm{S} \\
\mathrm{m}\end{array}$ & $\begin{array}{l}E \\
u\end{array}$ & $\begin{array}{l}G \\
\text { d }\end{array}$ & Tb & $\begin{array}{l}D \\
y\end{array}$ & $\begin{array}{l}\mathrm{H} \\
0\end{array}$ & $\mathrm{Er}$ & $\begin{array}{l}T \\
m\end{array}$ & $\mathbf{Y}$ & Lu & $\mathrm{Hf}$ & Th & U & $\begin{array}{l}\Sigma \\
R \\
E \\
E\end{array}$ & $\begin{array}{l}\text { (La } \\
\text { /Lu } \\
)_{N}\end{array}$ & $\begin{array}{l}(\mathrm{T} \\
\mathrm{h} / \\
\mathrm{U}) \\
N\end{array}$ & $\begin{array}{l}(\mathrm{L} \\
\mathrm{u} / \\
\mathrm{Hf}) \\
N\end{array}$ \\
\hline $\begin{array}{l}\mathrm{EM}^{1} \\
023\end{array}$ & $\begin{array}{l}4 . \\
3 \\
0\end{array}$ & $\begin{array}{l}0 . \\
06 \\
63\end{array}$ & $\begin{array}{l}1 \\
7\end{array}$ & $\begin{array}{l}0 . \\
1 \\
4 \\
1\end{array}$ & $\begin{array}{l}0 . \\
2 \\
1 \\
2\end{array}$ & $\begin{array}{l}0 . \\
03 \\
42\end{array}$ & $\begin{array}{l}0 . \\
15 \\
1\end{array}$ & $\begin{array}{l}0 . \\
03 \\
68\end{array}$ & $\begin{array}{l}0 . \\
01 \\
96\end{array}$ & $\begin{array}{l}0 . \\
04 \\
89\end{array}$ & $\begin{array}{l}0 . \\
00 \\
86\end{array}$ & $\begin{array}{l}0 . \\
05 \\
06\end{array}$ & $\begin{array}{l}0 . \\
01 \\
21\end{array}$ & $\begin{array}{l}0 . \\
03 \\
35\end{array}$ & $\begin{array}{l}0 \\
() \\
t\end{array}$ & $\begin{array}{l}n \\
i \\
2 i\end{array}$ & $\begin{array}{l}\text { J. } \\
00 \\
53\end{array}$ & $\begin{array}{l}0 . \\
02 \\
53\end{array}$ & $\begin{array}{l}0 . \\
01 \\
64\end{array}$ & $\begin{array}{l}0 . \\
01 \\
43\end{array}$ & $\begin{array}{l}0 . \\
7 \\
9 \\
1\end{array}$ & $\begin{array}{l}2.7 \\
9\end{array}$ & $\begin{array}{l}0 . \\
31\end{array}$ & $\begin{array}{l}0.9 \\
1\end{array}$ \\
\hline $\begin{array}{l}\text { EM } \\
089\end{array}$ & $\begin{array}{l}4 . \\
1 \\
1\end{array}$ & $\begin{array}{l}0 . \\
05 \\
59\end{array}$ & $\begin{array}{l}2 \\
8 \\
7\end{array}$ & $\begin{array}{l}0 . \\
1 \\
2 \\
2\end{array}$ & $\begin{array}{l}0 . \\
2 \\
2 \\
5\end{array}$ & $\begin{array}{l}0 . \\
03 \\
50\end{array}$ & $\begin{array}{l}0 . \\
15 \\
8\end{array}$ & $\begin{array}{l}0 . \\
04 \\
97\end{array}$ & $\begin{array}{l}0 . \\
02 \\
40\end{array}$ & $\begin{array}{l}0 . \\
06 \\
82\end{array}$ & $\begin{array}{l}0 . \\
01 \\
05\end{array}$ & $\begin{array}{l}0 . \\
07 \\
24\end{array}$ & $\begin{array}{l}0 . \\
01 \\
46\end{array}$ & $\begin{array}{l}0 . \\
30\end{array}$ & $\begin{array}{l}? \\
u \\
i 6\end{array}$ & $\begin{array}{l}0 . \\
04 \\
03\end{array}$ & $\begin{array}{l}0 . \\
00 \\
59\end{array}$ & $\begin{array}{l}0 . \\
02 \\
61\end{array}$ & $\begin{array}{l}0 . \\
09 \\
8\end{array}$ & $\begin{array}{l}0 . \\
00 \\
60\end{array}$ & $\begin{array}{l}0 . \\
8 \\
7 \\
4\end{array}$ & $\begin{array}{l}2.1 \\
7\end{array}$ & $\begin{array}{l}0 . \\
45\end{array}$ & $\begin{array}{l}0.9 \\
8\end{array}$ \\
\hline $\begin{array}{l}\text { EM } \\
090\end{array}$ & $\begin{array}{l}1 . \\
3 \\
8\end{array}$ & $\begin{array}{l}0 . \\
05 \\
95\end{array}$ & $\begin{array}{l}2 \\
9 \\
4 \\
\end{array}$ & $\begin{array}{l}0 . \\
0 \\
7 \\
0\end{array}$ & $\begin{array}{l}0 . \\
1 \\
7 \\
6\end{array}$ & $\begin{array}{l}0 . \\
02 \\
45\end{array}$ & $\begin{array}{l}0 . \\
11 \\
7\end{array}$ & $\begin{array}{l}0 . \\
03 \\
67\end{array}$ & $\begin{array}{l}0 . \\
01 \\
25\end{array}$ & $\begin{array}{l}0 . \\
04 \\
90\end{array}$ & $\begin{array}{l}0 . \\
00 \\
91\end{array}$ & $\begin{array}{l}0 . \\
05 \\
74\end{array}$ & $\begin{array}{l}0 . \\
01 \\
-\end{array}$ & $\begin{array}{l}0 . \\
03 \\
60\end{array}$ & $\begin{array}{l}0 . \\
i 0 \\
59\end{array}$ & $\begin{array}{l}0 . \\
03 \\
45\end{array}$ & $\begin{array}{l}0 . \\
00 \\
51\end{array}$ & $\begin{array}{l}0 . \\
01 \\
86\end{array}$ & $\begin{array}{l}0 . \\
00 \\
95\end{array}$ & $\begin{array}{l}0 . \\
00 \\
63\end{array}$ & $\begin{array}{l}0 . \\
6 \\
4 \\
7\end{array}$ & $\begin{array}{l}1.4 \\
4\end{array}$ & $\begin{array}{l}0 . \\
41\end{array}$ & $\begin{array}{l}1.1 \\
9\end{array}$ \\
\hline $\begin{array}{l}\text { EM } \\
091\end{array}$ & $\begin{array}{l}8 . \\
4 \\
7\end{array}$ & $\begin{array}{l}0 . \\
06 \\
69\end{array}$ & $\begin{array}{l}1 \\
0 \\
8\end{array}$ & $\begin{array}{l}0 . \\
5 \\
4 \\
2\end{array}$ & $\begin{array}{l}0 . \\
6 \\
0 \\
9\end{array}$ & $\begin{array}{l}0 . \\
13 \\
7\end{array}$ & $\begin{array}{l}0 . \\
57 \\
2\end{array}$ & $\begin{array}{l}0 . \\
13 \\
8\end{array}$ & $\begin{array}{l}0 . \\
05 \\
95\end{array}$ & $\begin{array}{l}0 . \\
16 \\
0\end{array}$ & $\begin{array}{l}0 . \\
02 \\
\end{array}$ & 3 & $\begin{array}{l}0 . \\
04\end{array}$ & $\begin{array}{l}0 . \\
08 \\
07\end{array}$ & $\begin{array}{l}0 . \\
01 \\
19\end{array}$ & $\begin{array}{l}0 . \\
07 \\
45\end{array}$ & $\begin{array}{l}0 . \\
01 \\
13\end{array}$ & $\begin{array}{l}0 . \\
02 \\
87\end{array}$ & $\begin{array}{l}0 . \\
02 \\
38\end{array}$ & $\begin{array}{l}0 . \\
04 \\
62\end{array}$ & $\begin{array}{l}2 . \\
5 \\
9\end{array}$ & $\begin{array}{l}5.1 \\
6\end{array}$ & $\begin{array}{l}0 . \\
14\end{array}$ & $\begin{array}{l}1.6 \\
5\end{array}$ \\
\hline $\begin{array}{l}\text { EM } \\
091 \\
\text { dup. }\end{array}$ & $\begin{array}{l}7 . \\
8 \\
1\end{array}$ & $\begin{array}{l}0 . \\
06 \\
21\end{array}$ & $\begin{array}{l}8 \\
6 \\
0\end{array}$ & $\begin{array}{l}0 . \\
4 \\
7 \\
5\end{array}$ & $\begin{array}{l}0 . \\
5 \\
3 \\
5\end{array}$ & $\begin{array}{l}0 . \\
12 \\
0\end{array}$ & $\begin{array}{l}0 . \\
49 \\
7\end{array}$ & $\begin{array}{l}0 . \\
11 \\
0\end{array}$ & $\begin{array}{l}0 . \\
04 \\
97\end{array}$ & $\begin{array}{l}0 . \\
14 \\
7\end{array}$ & $\begin{array}{l}0 . \\
i \\
8 s\end{array}$ & $\begin{array}{l}0 . \\
11 \\
5\end{array}$ & $\begin{array}{l}0 . \\
02 \\
59\end{array}$ & $\begin{array}{l}0 . \\
06 \\
91\end{array}$ & $\begin{array}{l}0 . \\
01 \\
11\end{array}$ & $\begin{array}{l}0 . \\
05 \\
95\end{array}$ & $\begin{array}{l}0 . \\
00 \\
94\end{array}$ & $\begin{array}{l}0 . \\
02 \\
11\end{array}$ & $\begin{array}{l}0 . \\
01 \\
67\end{array}$ & $\begin{array}{l}0 . \\
04 \\
52\end{array}$ & $\begin{array}{l}2 . \\
2 \\
4\end{array}$ & $\begin{array}{l}5.5 \\
2\end{array}$ & $\begin{array}{l}0 . \\
10\end{array}$ & $\begin{array}{l}1.8 \\
6\end{array}$ \\
\hline $\begin{array}{l}\text { EM } \\
111\end{array}$ & $\begin{array}{l}3 . \\
3 \\
3\end{array}$ & $\begin{array}{l}0 . \\
05 \\
44\end{array}$ & $\begin{array}{l}3 \\
2 \\
3\end{array}$ & $\begin{array}{l}0 . \\
1 \\
6 \\
6\end{array}$ & $\begin{array}{l}0 . \\
3 \\
2 \\
6\end{array}$ & $\begin{array}{l}0 . \\
04 \\
73\end{array}$ & $\begin{array}{l}0 . \\
20 \\
1\end{array}$ & $\begin{array}{l}0 . \\
05 \\
42\end{array}$ & $\begin{array}{l}0 . \\
02 \\
38\end{array}$ & ¿06 & $\begin{array}{l}0 . \\
01 \\
16\end{array}$ & $\begin{array}{l}0 . \\
07 \\
15\end{array}$ & $\begin{array}{l}0 . \\
01 \\
61\end{array}$ & $\begin{array}{l}0 . \\
04 \\
68\end{array}$ & $\begin{array}{l}0 . \\
00 \\
70\end{array}$ & $\begin{array}{l}0 . \\
04 \\
39\end{array}$ & $\begin{array}{l}0 . \\
00 \\
67\end{array}$ & $\begin{array}{l}0 . \\
02 \\
38\end{array}$ & $\begin{array}{l}0 . \\
01 \\
66\end{array}$ & $\begin{array}{l}0 . \\
01 \\
27\end{array}$ & $\begin{array}{l}1 . \\
0 \\
9\end{array}$ & $\begin{array}{l}2.5 \\
8\end{array}$ & $\begin{array}{l}0 . \\
35\end{array}$ & $\begin{array}{l}1.2 \\
3\end{array}$ \\
\hline $\begin{array}{l}\text { EM } \\
120\end{array}$ & $\begin{array}{l}2 . \\
8 \\
9\end{array}$ & $\begin{array}{l}0 . \\
07 \\
11\end{array}$ & $\begin{array}{l}2 \\
9 \\
2 \\
2\end{array}$ & $\begin{array}{l}0 . \\
3 \\
7 \\
1\end{array}$ & $\begin{array}{l}0 . \\
4 \\
1 \\
9\end{array}$ & $\begin{array}{l}0 . \\
08 \\
30\end{array}$ & $\begin{array}{l}0 . \\
33 \\
7\end{array}$ & $\begin{array}{l}0 . \\
07 \\
0 \varepsilon\end{array}$ & $\begin{array}{l}n . \\
u_{2} \\
81\end{array}$ & $\begin{array}{l}0 \\
u y \\
04\end{array}$ & $\begin{array}{l}0 . \\
01 \\
38\end{array}$ & $\begin{array}{l}0 . \\
07 \\
79\end{array}$ & $\begin{array}{l}0 . \\
01 \\
78\end{array}$ & $\begin{array}{l}0 . \\
05 \\
07\end{array}$ & $\begin{array}{l}0 . \\
00 \\
70\end{array}$ & $\begin{array}{l}0 . \\
04 \\
29\end{array}$ & $\begin{array}{l}0 . \\
00 \\
73\end{array}$ & $\begin{array}{l}0 . \\
04 \\
35\end{array}$ & $\begin{array}{l}0 . \\
02 \\
82\end{array}$ & $\begin{array}{l}0 . \\
02 \\
05\end{array}$ & $\begin{array}{l}1 . \\
6 \\
2\end{array}$ & $\begin{array}{l}5.5 \\
5\end{array}$ & $\begin{array}{l}0 . \\
36\end{array}$ & $\begin{array}{l}0.7 \\
1\end{array}$ \\
\hline $\begin{array}{l}\text { EM } \\
120 \\
\text { dup. }\end{array}$ & $\begin{array}{l}3 . \\
3 \\
6\end{array}$ & $\begin{array}{l}0 . \\
06 \\
66\end{array}$ & $\begin{array}{l}3 \\
3\end{array}$ & $\begin{array}{l}0 . \\
4 \\
4 \\
2\end{array}$ & $\begin{array}{l}0 . \\
4 \\
8 \\
3\end{array}$ & $\begin{array}{l}0 . \\
09 \\
66\end{array}$ & $\begin{array}{l}0 . \\
40 \\
0\end{array}$ & $\begin{array}{l}0 b \\
0\end{array}$ & $\begin{array}{l}0 . \\
03 \\
28\end{array}$ & $\begin{array}{l}0 . \\
11 \\
1\end{array}$ & $\begin{array}{l}0 . \\
01 \\
64\end{array}$ & $\begin{array}{l}0 . \\
09 \\
90\end{array}$ & $\begin{array}{l}0 . \\
02 \\
15\end{array}$ & $\begin{array}{l}0 . \\
05 \\
95\end{array}$ & $\begin{array}{l}0 . \\
00 \\
80\end{array}$ & $\begin{array}{l}0 . \\
05 \\
02\end{array}$ & $\begin{array}{l}0 . \\
00 \\
95\end{array}$ & $\begin{array}{l}0 . \\
02 \\
32\end{array}$ & $\begin{array}{l}0 . \\
02 \\
36\end{array}$ & $\begin{array}{l}0 . \\
02 \\
32\end{array}$ & $\begin{array}{l}1 . \\
9 \\
1\end{array}$ & $\begin{array}{l}5.1 \\
4\end{array}$ & $\begin{array}{l}0 . \\
28\end{array}$ & $\begin{array}{l}1.7 \\
0\end{array}$ \\
\hline $\begin{array}{l}\text { Kerma } \\
\text { n } 009\end{array}$ & $\begin{array}{l}2 \\
9 . \\
4\end{array}$ & $\begin{array}{l}0 . \\
07 \\
36\end{array}$ & $\begin{array}{l}1 \\
2 \\
7\end{array}$ & $\begin{array}{l}0 . \\
0 \\
8 \\
7\end{array}$ & $\begin{array}{l}0 . \\
2 \\
0 \\
2\end{array}$ & $\begin{array}{l}0 . \\
02 \\
6\end{array}$ & $\begin{array}{l}0 . \\
3\end{array}$ & $\begin{array}{l}n \\
\text { U3 } \\
33\end{array}$ & $\begin{array}{l}0 . \\
01 \\
48\end{array}$ & $\begin{array}{l}0 . \\
04 \\
86\end{array}$ & $\begin{array}{l}0 . \\
00 \\
90\end{array}$ & $\begin{array}{l}0 . \\
05 \\
11\end{array}$ & $\begin{array}{l}0 . \\
01 \\
21\end{array}$ & $\begin{array}{l}0 . \\
03 \\
57\end{array}$ & $\begin{array}{l}0 . \\
00 \\
49\end{array}$ & $\begin{array}{l}0 . \\
03 \\
36\end{array}$ & $\begin{array}{l}0 . \\
00 \\
62\end{array}$ & $\begin{array}{l}0 . \\
02 \\
56\end{array}$ & $\begin{array}{l}0 . \\
02 \\
10\end{array}$ & $\begin{array}{l}0 . \\
06 \\
26\end{array}$ & $\begin{array}{l}0 . \\
6 \\
7 \\
7\end{array}$ & $\begin{array}{l}1.4 \\
6\end{array}$ & $\begin{array}{l}0 . \\
09\end{array}$ & $\begin{array}{l}1.0 \\
5\end{array}$ \\
\hline $\begin{array}{l}\text { Kerma } \\
\text { n } 013\end{array}$ & $\begin{array}{l}2 \\
2 . \\
4\end{array}$ & $\begin{array}{l}0 . \\
06 \\
36\end{array}$ & $\begin{array}{l}7 \\
2 \\
2\end{array}$ & $\begin{array}{l}0 . \\
0 \\
6 \\
3\end{array}$ & $\begin{array}{l}- \\
0 . \\
1 \\
5 \\
4\end{array}$ & $\begin{array}{l}01 \\
01 \\
97\end{array}$ & $\begin{array}{l}U . \\
09 \\
93\end{array}$ & $\begin{array}{l}0 . \\
03 \\
13\end{array}$ & $\begin{array}{l}0 . \\
01 \\
23\end{array}$ & $\begin{array}{l}0 . \\
03 \\
88\end{array}$ & $\begin{array}{l}0 . \\
00 \\
71\end{array}$ & $\begin{array}{l}0 . \\
04 \\
85\end{array}$ & $\begin{array}{l}0 . \\
01 \\
08\end{array}$ & $\begin{array}{l}0 . \\
03 \\
07\end{array}$ & $\begin{array}{l}0 . \\
00 \\
53\end{array}$ & $\begin{array}{l}0 . \\
03 \\
16\end{array}$ & $\begin{array}{l}0 . \\
00 \\
51\end{array}$ & $\begin{array}{l}0 . \\
02 \\
39\end{array}$ & $\begin{array}{l}0 . \\
01 \\
40\end{array}$ & $\begin{array}{l}0 . \\
04 \\
07\end{array}$ & $\begin{array}{l}0 . \\
5 \\
5 \\
8\end{array}$ & $\begin{array}{l}1.2 \\
9\end{array}$ & $\begin{array}{l}0 . \\
09\end{array}$ & $\begin{array}{l}0.9 \\
4\end{array}$ \\
\hline $\begin{array}{l}\text { Kerma } \\
\text { n } 026\end{array}$ & $\begin{array}{l}5 . \\
7 \\
4\end{array}$ & $\begin{array}{l}0 . \\
06 \\
40\end{array}$ & $\begin{array}{l}4 \\
\\
9 \\
1\end{array}$ & $\begin{array}{l}0 . \\
0 \\
4 \\
0\end{array}$ & $\begin{array}{l}0 . \\
1 \\
1 \\
0\end{array}$ & $\begin{array}{l}0 . \\
01 \\
43\end{array}$ & $\begin{array}{l}0 . \\
06 \\
81\end{array}$ & $\begin{array}{l}0 . \\
02 \\
74\end{array}$ & $\begin{array}{l}0 . \\
01 \\
20\end{array}$ & $\begin{array}{l}0 . \\
03 \\
64\end{array}$ & $\begin{array}{l}0 . \\
00 \\
63\end{array}$ & $\begin{array}{l}0 . \\
04 \\
19\end{array}$ & $\begin{array}{l}0 . \\
01 \\
07\end{array}$ & $\begin{array}{l}0 . \\
02 \\
94\end{array}$ & $\begin{array}{l}0 . \\
00 \\
45\end{array}$ & $\begin{array}{l}0 . \\
03 \\
18\end{array}$ & $\begin{array}{l}0 . \\
00 \\
44\end{array}$ & $\begin{array}{l}0 . \\
02 \\
87\end{array}$ & $\begin{array}{l}0 . \\
00 \\
88\end{array}$ & $\begin{array}{l}0 . \\
01 \\
77\end{array}$ & $\begin{array}{l} \\
\\
4 \\
3 \\
7\end{array}$ & $\begin{array}{l}1.0 \\
5\end{array}$ & $\begin{array}{l}0 . \\
14\end{array}$ & $\begin{array}{l}0.6 \\
0\end{array}$ \\
\hline $\begin{array}{l}\text { Kerma } \\
\text { n } 026 \\
\text { dup. }\end{array}$ & $\begin{array}{l}5 . \\
7 \\
4\end{array}$ & $\begin{array}{l}0 . \\
06 \\
36\end{array}$ & $\begin{array}{l}4 \\
7 \\
1 \\
\end{array}$ & $\begin{array}{l}0 . \\
0 \\
4 \\
1 \\
\end{array}$ & $\begin{array}{l}0 . \\
1 \\
0 \\
4\end{array}$ & $\begin{array}{l}0 . \\
01 \\
67\end{array}$ & $\begin{array}{l}0 . \\
07 \\
53\end{array}$ & $\begin{array}{l}0 . \\
02 \\
66\end{array}$ & $\begin{array}{l}0 . \\
01 \\
20\end{array}$ & $\begin{array}{l}0 . \\
03 \\
66\end{array}$ & $\begin{array}{l}0 . \\
00 \\
72\end{array}$ & $\begin{array}{l}0 . \\
04 \\
46\end{array}$ & $\begin{array}{l}0 . \\
01 \\
01\end{array}$ & $\begin{array}{l}0 . \\
03 \\
22\end{array}$ & $\begin{array}{l}0 . \\
00 \\
47\end{array}$ & $\begin{array}{l}0 . \\
03 \\
18\end{array}$ & $\begin{array}{l}0 . \\
00 \\
51\end{array}$ & $\begin{array}{l}0 . \\
02 \\
22\end{array}$ & $\begin{array}{l}0 . \\
00 \\
74\end{array}$ & $\begin{array}{l}0 . \\
01 \\
87\end{array}$ & $\begin{array}{l}0 . \\
4 \\
4 \\
8\end{array}$ & $\begin{array}{l}0.8 \\
6\end{array}$ & $\begin{array}{l}0 . \\
10\end{array}$ & $\begin{array}{l}0.9 \\
9\end{array}$ \\
\hline $\begin{array}{l}A^{2} \\
09135\end{array}$ & $\begin{array}{l}9 . \\
5 \\
4\end{array}$ & $\begin{array}{l}0 . \\
32 \\
3\end{array}$ & $\begin{array}{l}3 \\
0 \\
7\end{array}$ & $\begin{array}{l}0 . \\
3 \\
0 \\
5\end{array}$ & $\begin{array}{l}0 . \\
7 \\
5 \\
2\end{array}$ & $\begin{array}{l}0 . \\
11 \\
8\end{array}$ & $\begin{array}{l}0 . \\
55 \\
5\end{array}$ & $\begin{array}{l}0 . \\
17 \\
1\end{array}$ & $\begin{array}{l}0 . \\
07 \\
03\end{array}$ & $\begin{array}{l}0 . \\
24 \\
7\end{array}$ & $\begin{array}{l}0 . \\
04 \\
65\end{array}$ & $\begin{array}{l}0 . \\
29 \\
8\end{array}$ & $\begin{array}{l}0 . \\
07 \\
05\end{array}$ & $\begin{array}{l}0 . \\
19 \\
7\end{array}$ & $\begin{array}{l}0 . \\
02 \\
79\end{array}$ & $\begin{array}{l}0 . \\
20 \\
5\end{array}$ & $\begin{array}{l}0 . \\
03 \\
74\end{array}$ & $\begin{array}{l}0 . \\
13 \\
9\end{array}$ & $\begin{array}{l}0 . \\
05 \\
10\end{array}$ & $\begin{array}{l}0 . \\
00 \\
94\end{array}$ & $\begin{array}{l}3 . \\
1 \\
0\end{array}$ & $\begin{array}{l}0.8 \\
5\end{array}$ & $\begin{array}{l}1 . \\
48\end{array}$ & $\begin{array}{l}1.1 \\
7\end{array}$ \\
\hline $\begin{array}{l}\text { A } \\
09387\end{array}$ & $\begin{array}{l}8 . \\
4 \\
6\end{array}$ & $\begin{array}{l}0 . \\
35 \\
1\end{array}$ & $\begin{array}{l}2 \\
7 \\
2\end{array}$ & $\begin{array}{l}0 . \\
2 \\
8 \\
1\end{array}$ & $\begin{array}{l}0 . \\
6 \\
6 \\
6\end{array}$ & $\begin{array}{l}0 . \\
10 \\
1\end{array}$ & $\begin{array}{l}0 . \\
51 \\
9\end{array}$ & $\begin{array}{l}0 . \\
18 \\
1\end{array}$ & $\begin{array}{l}0 . \\
06 \\
43\end{array}$ & $\begin{array}{l}0 . \\
22 \\
7\end{array}$ & $\begin{array}{l}0 . \\
03 \\
85\end{array}$ & $\begin{array}{l}0 . \\
29 \\
3\end{array}$ & $\begin{array}{l}0 . \\
05 \\
82\end{array}$ & $\begin{array}{l}0 . \\
18 \\
5\end{array}$ & $\begin{array}{l}0 . \\
02 \\
55\end{array}$ & $\begin{array}{l}0 . \\
18 \\
2\end{array}$ & $\begin{array}{l}0 . \\
02 \\
45\end{array}$ & $\begin{array}{l}0 . \\
11 \\
9\end{array}$ & $\begin{array}{l}0 . \\
03 \\
4 .\end{array}$ & $\begin{array}{l}0 . \\
01 \\
02\end{array}$ & $\begin{array}{l}2 . \\
8 \\
5\end{array}$ & $\begin{array}{l}1.2 \\
0\end{array}$ & $\begin{array}{l}0 . \\
91\end{array}$ & $\begin{array}{l}0.8 \\
9\end{array}$ \\
\hline $\begin{array}{l}\text { A } \\
09436\end{array}$ & $\begin{array}{l}6 . \\
4 \\
2\end{array}$ & $\begin{array}{l}0 . \\
31 \\
3\end{array}$ & $\begin{array}{l}1 \\
1 \\
3\end{array}$ & $\begin{array}{l}0 . \\
1 \\
4 \\
7\end{array}$ & $\begin{array}{l}0 . \\
3 \\
2 \\
7\end{array}$ & $\begin{array}{l}0 . \\
05 \\
17\end{array}$ & $\begin{array}{l}0 . \\
24 \\
0\end{array}$ & $\begin{array}{l}0 . \\
09 \\
48\end{array}$ & $\begin{array}{l}0 . \\
03 \\
14\end{array}$ & $\begin{array}{l}0 . \\
12 \\
9\end{array}$ & $\begin{array}{l}0 . \\
02 \\
33\end{array}$ & $\begin{array}{l}0 . \\
14 \\
5\end{array}$ & $\begin{array}{l}0 . \\
03 \\
10\end{array}$ & $\begin{array}{l}0 . \\
09 \\
88\end{array}$ & $\begin{array}{l}0 . \\
01 \\
23\end{array}$ & $\begin{array}{l}0 . \\
09 \\
67\end{array}$ & $\begin{array}{l}0 . \\
01 \\
18\end{array}$ & $\begin{array}{l}0 . \\
04 \\
2\end{array}$ & $\begin{array}{l}0 . \\
00 \\
89\end{array}$ & $\begin{array}{l}0 . \\
00 \\
98\end{array}$ & $\begin{array}{l}1 . \\
4 \\
4\end{array}$ & $\begin{array}{l}1.3 \\
0\end{array}$ & $\begin{array}{l}0 . \\
25\end{array}$ & $\begin{array}{l}1.2 \\
2\end{array}$ \\
\hline $\begin{array}{l}\text { A } \\
09455\end{array}$ & $\begin{array}{l}9 . \\
8 \\
0\end{array}$ & $\begin{array}{l}0 . \\
37 \\
7\end{array}$ & $\begin{array}{l}3 \\
2 \\
0\end{array}$ & $\begin{array}{l}0 . \\
3 \\
3 \\
6\end{array}$ & $\begin{array}{l}0 . \\
7 \\
9 \\
1\end{array}$ & $\begin{array}{l}0 . \\
13 \\
2\end{array}$ & $\begin{array}{l}0 . \\
59 \\
5\end{array}$ & $\begin{array}{l}0 . \\
21 \\
0\end{array}$ & $\begin{array}{l}0 . \\
07 \\
24\end{array}$ & $\begin{array}{l}0 . \\
29 \\
5\end{array}$ & $\begin{array}{l}0 . \\
04 \\
49\end{array}$ & $\begin{array}{l}0 . \\
32 \\
9\end{array}$ & $\begin{array}{l}0 . \\
07 \\
53\end{array}$ & $\begin{array}{l}0 . \\
20 \\
9\end{array}$ & $\begin{array}{l}0 . \\
03 \\
47\end{array}$ & $\begin{array}{l}0 . \\
22 \\
9\end{array}$ & $\begin{array}{l}0 . \\
02 \\
92\end{array}$ & $\begin{array}{l}0 . \\
14 \\
0\end{array}$ & $\begin{array}{l}0 . \\
03 \\
44\end{array}$ & $\begin{array}{l}0 . \\
01 \\
10\end{array}$ & $\begin{array}{l}3 . \\
3 \\
9\end{array}$ & $\begin{array}{l}1.2 \\
0\end{array}$ & $\begin{array}{l}0 . \\
85\end{array}$ & $\begin{array}{l}0.9 \\
1\end{array}$ \\
\hline $\begin{array}{l}\text { A } \\
09516\end{array}$ & $\begin{array}{l}7 . \\
2 \\
7\end{array}$ & $\begin{array}{l}0 . \\
33 \\
5\end{array}$ & $\begin{array}{l}2 \\
9 \\
6\end{array}$ & $\begin{array}{l}0 . \\
2 \\
7 \\
9\end{array}$ & $\begin{array}{l}0 . \\
6 \\
2 \\
0\end{array}$ & $\begin{array}{l}0 . \\
09 \\
27\end{array}$ & $\begin{array}{l}0 . \\
46 \\
0\end{array}$ & $\begin{array}{l}0 . \\
13 \\
1\end{array}$ & $\begin{array}{l}0 . \\
05 \\
19\end{array}$ & $\begin{array}{l}0 . \\
20 \\
6\end{array}$ & $\begin{array}{l}0 . \\
03 \\
25\end{array}$ & $\begin{array}{l}0 . \\
22 \\
5\end{array}$ & $\begin{array}{l}0 . \\
05 \\
40\end{array}$ & $\begin{array}{l}0 . \\
16 \\
5\end{array}$ & $\begin{array}{l}0 . \\
02 \\
23\end{array}$ & $\begin{array}{l}0 . \\
14 \\
9\end{array}$ & $\begin{array}{l}0 . \\
02 \\
51\end{array}$ & $\begin{array}{l}0 . \\
11 \\
0\end{array}$ & $\begin{array}{l}0 . \\
03 \\
85\end{array}$ & $\begin{array}{l}0 . \\
01 \\
50\end{array}$ & $\begin{array}{l}2 . \\
5 \\
1\end{array}$ & $\begin{array}{l}1.1 \\
6\end{array}$ & $\begin{array}{l}0 . \\
70\end{array}$ & $\begin{array}{l}1.0 \\
0\end{array}$ \\
\hline
\end{tabular}




\begin{tabular}{|c|c|c|c|c|c|c|c|c|c|c|c|c|c|c|c|c|c|c|c|c|c|c|c|c|}
\hline $\begin{array}{l}\text { A } \\
09618\end{array}$ & $\begin{array}{l}8 . \\
3 \\
9\end{array}$ & $\begin{array}{l}0 . \\
34 \\
7\end{array}$ & $\begin{array}{l}3 \\
2 \\
8\end{array}$ & $\begin{array}{l}0 . \\
4 \\
2 \\
2\end{array}$ & $\begin{array}{l}0 . \\
8 \\
6 \\
7\end{array}$ & $\begin{array}{l}0 . \\
12 \\
3\end{array}$ & $\begin{array}{l}0 . \\
61 \\
0\end{array}$ & $\begin{array}{l}0 . \\
18 \\
5\end{array}$ & $\begin{array}{l}0 . \\
06 \\
33\end{array}$ & $\begin{array}{l}0 . \\
25 \\
1\end{array}$ & $\begin{array}{l}0 . \\
03 \\
92\end{array}$ & $\begin{array}{l}0 . \\
29 \\
7\end{array}$ & $\begin{array}{l}0 . \\
06 \\
53\end{array}$ & $\begin{array}{l}0 . \\
20 \\
6\end{array}$ & $\begin{array}{l}0 . \\
02 \\
87\end{array}$ & $\begin{array}{l}0 . \\
19 \\
9\end{array}$ & $\begin{array}{l}0 . \\
03 \\
08\end{array}$ & $\begin{array}{l}0 . \\
11 \\
8\end{array}$ & $\begin{array}{l}0 . \\
03 \\
82\end{array}$ & $\begin{array}{l}0 . \\
02 \\
89\end{array}$ & $\begin{array}{l}3 . \\
3 \\
9\end{array}$ & $\begin{array}{l}1.4 \\
3\end{array}$ & $\begin{array}{l}0 . \\
36\end{array}$ & $\begin{array}{l}1.1 \\
4\end{array}$ \\
\hline $\begin{array}{l}\text { A } \\
10091\end{array}$ & $\begin{array}{l}8 . \\
5 \\
9\end{array}$ & $\begin{array}{l}0 . \\
38 \\
4\end{array}$ & $\begin{array}{l}1 \\
5 \\
2 \\
\end{array}$ & $\begin{array}{l}0 . \\
1 \\
9 \\
3 \\
\end{array}$ & $\begin{array}{l}0 . \\
4 \\
3 \\
4 \\
\end{array}$ & $\begin{array}{l}0 . \\
07 \\
45\end{array}$ & $\begin{array}{l}0 . \\
40 \\
2\end{array}$ & $\begin{array}{l}0 . \\
12 \\
6\end{array}$ & $\begin{array}{l}0 . \\
05 \\
37\end{array}$ & $\begin{array}{l}0 . \\
16 \\
5\end{array}$ & $\begin{array}{l}0 . \\
02 \\
83\end{array}$ & $\begin{array}{l}0 . \\
20 \\
5\end{array}$ & $\begin{array}{l}0 . \\
04 \\
36\end{array}$ & $\begin{array}{l}0 . \\
12 \\
9\end{array}$ & $\begin{array}{l}0 . \\
01 \\
93\end{array}$ & $\begin{array}{l}0 . \\
11 \\
4\end{array}$ & $\begin{array}{l}0 . \\
01 \\
57\end{array}$ & $\begin{array}{l}0 . \\
04 \\
73\end{array}$ & $\begin{array}{l}0 . \\
01 \\
07\end{array}$ & $\begin{array}{l}0 . \\
01 \\
37\end{array}$ & $\begin{array}{l}2 . \\
0 \\
0\end{array}$ & $\begin{array}{l}1.2 \\
9\end{array}$ & $\begin{array}{l}0 . \\
21\end{array}$ & $\begin{array}{l}1.4 \\
4\end{array}$ \\
\hline $\begin{array}{l}\text { A } \\
10182\end{array}$ & $\begin{array}{l}8 . \\
9 \\
0\end{array}$ & $\begin{array}{l}0 . \\
34 \\
4\end{array}$ & $\begin{array}{l}2 \\
4 \\
8 \\
\end{array}$ & $\begin{array}{l}0 . \\
2 \\
4 \\
7 \\
\end{array}$ & $\begin{array}{l}0 . \\
6 \\
8 \\
1 \\
\end{array}$ & $\begin{array}{l}0 . \\
10 \\
7\end{array}$ & $\begin{array}{l}0 . \\
48 \\
5\end{array}$ & $\begin{array}{l}0 . \\
15 \\
5\end{array}$ & $\begin{array}{l}0 . \\
05 \\
87\end{array}$ & $\begin{array}{l}0 . \\
23 \\
2\end{array}$ & $\begin{array}{l}0 . \\
04 \\
17\end{array}$ & $\begin{array}{l}0 . \\
28 \\
1\end{array}$ & $\begin{array}{l}0 . \\
06 \\
11\end{array}$ & $\begin{array}{l}0 . \\
18 \\
7\end{array}$ & $\begin{array}{l}0 . \\
02 \\
84\end{array}$ & $\begin{array}{l}0 . \\
19 \\
0\end{array}$ & $\begin{array}{l}0 . \\
03 \\
08\end{array}$ & $\begin{array}{l}0 . \\
11 \\
9\end{array}$ & $\begin{array}{l}0 . \\
03 \\
74\end{array}$ & $\begin{array}{l}0 . \\
01 \\
10\end{array}$ & $\begin{array}{l}2 . \\
7 \\
9\end{array}$ & $\begin{array}{l}0.8 \\
4\end{array}$ & $\begin{array}{l}0 . \\
92\end{array}$ & $\begin{array}{l}1.1 \\
2\end{array}$ \\
\hline $\begin{array}{l}\text { A } \\
10187\end{array}$ & $\begin{array}{l}9 . \\
4 \\
5\end{array}$ & $\begin{array}{l}0 . \\
35 \\
3\end{array}$ & $\begin{array}{l}3 \\
5 \\
5 \\
\end{array}$ & $\begin{array}{l}0 . \\
3 \\
0 \\
3 \\
\end{array}$ & $\begin{array}{l}0 . \\
7 \\
2 \\
8 \\
\end{array}$ & $\begin{array}{l}0 . \\
11 \\
3\end{array}$ & $\begin{array}{l}0 . \\
53 \\
1\end{array}$ & $\begin{array}{l}0 . \\
16 \\
4\end{array}$ & $\begin{array}{l}0 . \\
06 \\
98\end{array}$ & $\begin{array}{l}0 . \\
22 \\
8\end{array}$ & $\begin{array}{l}0 . \\
04 \\
24\end{array}$ & $\begin{array}{l}0 . \\
28 \\
9\end{array}$ & $\begin{array}{l}0 . \\
06 \\
71\end{array}$ & $\begin{array}{l}0 . \\
18 \\
8\end{array}$ & $\begin{array}{l}0 . \\
02 \\
77\end{array}$ & $\begin{array}{l}0 . \\
20 \\
3\end{array}$ & $\begin{array}{l}0 . \\
02 \\
88\end{array}$ & $\begin{array}{l}0 . \\
12 \\
6\end{array}$ & $\begin{array}{l}0 . \\
04 \\
60\end{array}$ & $\begin{array}{l}0 . \\
01 \\
28\end{array}$ & $\begin{array}{l}2 . \\
9 \\
8\end{array}$ & $\begin{array}{l}1.1 \\
0\end{array}$ & $\begin{array}{l}0 . \\
98\end{array}$ & $\begin{array}{l}0.9 \\
9\end{array}$ \\
\hline $\begin{array}{l}\text { A } \\
10224\end{array}$ & $\begin{array}{l}8 . \\
2 \\
6\end{array}$ & $\begin{array}{l}0 . \\
30 \\
6\end{array}$ & $\begin{array}{l}2 \\
4 \\
8\end{array}$ & $\begin{array}{l}0 . \\
2 \\
4 \\
7\end{array}$ & $\begin{array}{l}0 . \\
5 \\
8 \\
6 \\
\end{array}$ & $\begin{array}{l}0 . \\
09 \\
05\end{array}$ & $\begin{array}{l}0 . \\
42 \\
8\end{array}$ & $\begin{array}{l}0 \\
14 \\
2\end{array}$ & $\begin{array}{l}0 . \\
05 \\
55\end{array}$ & $\begin{array}{l}0 . \\
19 \\
9\end{array}$ & $\begin{array}{l}0 . \\
03 \\
91\end{array}$ & $\begin{array}{l}0 . \\
25 \\
7\end{array}$ & $\begin{array}{l}0 . \\
05 \\
56\end{array}$ & $\begin{array}{l}0 . \\
17 \\
2\end{array}$ & $\begin{array}{l}0 . \\
02 \\
37\end{array}$ & $\begin{array}{l}0 . \\
18 \\
4\end{array}$ & $\begin{array}{l}0 . \\
02 \\
0\end{array}$ & $\begin{array}{l}0 . \\
10 \\
6\end{array}$ & $\begin{array}{l}0 . \\
02 \\
81\end{array}$ & $\begin{array}{l}0 . \\
01 \\
17\end{array}$ & $\begin{array}{l}2 . \\
5 \\
0\end{array}$ & $\begin{array}{l}1.2 \\
4\end{array}$ & $\begin{array}{l}0 . \\
65\end{array}$ & $\begin{array}{l}0.8 \\
6\end{array}$ \\
\hline $\begin{array}{l}\text { A } \\
12056\end{array}$ & $\begin{array}{l}7 . \\
4 \\
4\end{array}$ & $\begin{array}{l}0 . \\
38 \\
8\end{array}$ & $\begin{array}{l}1 \\
7 \\
6 \\
\end{array}$ & $\begin{array}{l}0 . \\
2 \\
8 \\
7\end{array}$ & $\begin{array}{l}0 . \\
6 \\
5 \\
0 \\
\end{array}$ & $\begin{array}{l}0 . \\
10 \\
9\end{array}$ & $\begin{array}{l}0 . \\
53 \\
9\end{array}$ & $\begin{array}{l}0 . \\
15 \\
6\end{array}$ & $\begin{array}{l}0 . \\
05 \\
59\end{array}$ & $\begin{array}{l}0 . \\
23 \\
1\end{array}$ & $\begin{array}{l}0 . \\
03 \\
76\end{array}$ & $\begin{array}{l}0 . \\
27 \\
4\end{array}$ & $\begin{array}{l}0 . \\
06 \\
14\end{array}$ & $\begin{array}{l}0 . \\
17 \\
7\end{array}$ & $\begin{array}{l}0 . \\
03 \\
13\end{array}$ & $\begin{array}{l}0 . \\
18 \\
3\end{array}$ & $\begin{array}{l}0 . \\
02 \\
88\end{array}$ & $\begin{array}{l}0 . \\
08 \\
67\end{array}$ & $\begin{array}{l}0 . \\
01 \\
57\end{array}$ & $\begin{array}{l}0 . \\
01 \\
34\end{array}$ & $\begin{array}{l}2 . \\
8 \\
2\end{array}$ & $\begin{array}{l}1.0 \\
4\end{array}$ & $\begin{array}{l}0 . \\
32\end{array}$ & $\begin{array}{l}1.4 \\
4\end{array}$ \\
\hline $\begin{array}{l}\text { Mean } \\
\mathrm{H}\end{array}$ & $\begin{array}{l}1 \\
0 . \\
0 \\
0 \\
\end{array}$ & $\begin{array}{l}0 . \\
36\end{array}$ & $\begin{array}{l}4 \\
2 \\
0 \\
\end{array}$ & $\begin{array}{l}0 . \\
2 \\
9 \\
5\end{array}$ & $\begin{array}{l}0 . \\
8 \\
3 \\
0 \\
\end{array}$ & $\begin{array}{l}0 . \\
12 \\
3\end{array}$ & $\begin{array}{l}0 . \\
62 \\
8\end{array}$ & $\begin{array}{l}0 . \\
18 \\
5\end{array}$ & $\begin{array}{l}0 . \\
07 \\
3\end{array}$ & $\begin{array}{l}0 . \\
29 \\
9\end{array}$ & $\begin{array}{l}0 . \\
05 \\
3\end{array}$ & $\begin{array}{l}0 . \\
34 \\
3\end{array}$ & $\begin{array}{l}0 . \\
07 \\
3\end{array}$ & $\begin{array}{l}0 . \\
22 \\
6\end{array}$ & $\begin{array}{l}0 . \\
03 \\
9\end{array}$ & $\begin{array}{l}0 . \\
2 C \\
5\end{array}$ & $\begin{array}{l}0 . \\
03 \\
1\end{array}$ & $\begin{array}{l}0 . \\
18 \\
0\end{array}$ & $\begin{array}{l}0 . \\
04 \\
2\end{array}$ & $\begin{array}{l}0 . \\
01 \\
2\end{array}$ & $\begin{array}{l}3 . \\
4 \\
0\end{array}$ & $\begin{array}{l}1.0 \\
0\end{array}$ & $\begin{array}{l}0 . \\
95\end{array}$ & $\begin{array}{l}0.7 \\
5\end{array}$ \\
\hline $\begin{array}{l}\text { Mean } \\
\mathrm{L}\end{array}$ & $\begin{array}{l}1 \\
1 . \\
1 \\
0\end{array}$ & $\begin{array}{l}0 . \\
39\end{array}$ & $\begin{array}{l}3 \\
7 \\
0 \\
\end{array}$ & $\begin{array}{l}0 . \\
3 \\
1 \\
0\end{array}$ & $\begin{array}{l}0 . \\
9 \\
0 \\
0\end{array}$ & $\begin{array}{l}0 . \\
13 \\
2\end{array}$ & $\begin{array}{l}0 . \\
68 \\
2\end{array}$ & $\begin{array}{l}0 . \\
19 \\
5\end{array}$ & $\begin{array}{l}0 . \\
07 \\
8\end{array}$ & $\begin{array}{l}0 . \\
31 \\
0\end{array}$ & $\begin{array}{l}0 . \\
05 \\
7\end{array}$ & $\begin{array}{l}0 . \\
36 \\
6\end{array}$ & $\begin{array}{l}0 . \\
08 \\
1\end{array}$ & $\begin{array}{l}0 . \\
24 \\
8\end{array}$ & $\begin{array}{l}0 . \\
03 \\
9\end{array}$ & ? & $\begin{array}{l}0 . \\
3 \\
3\end{array}$ & $\begin{array}{l}0 . \\
17 \\
0\end{array}$ & $\begin{array}{l}0 . \\
04 \\
3\end{array}$ & $\begin{array}{l}0 . \\
01 \\
3\end{array}$ & $\begin{array}{l}3 . \\
6 \\
5\end{array}$ & $\begin{array}{l}0.9 \\
8\end{array}$ & $\begin{array}{l}0 . \\
90\end{array}$ & $\begin{array}{l}0.8 \\
4\end{array}$ \\
\hline $\begin{array}{l}\text { Mean } \\
\text { LL }\end{array}$ & $\begin{array}{l}1 \\
1 . \\
1 \\
0\end{array}$ & $\begin{array}{l}0 . \\
37\end{array}$ & $\begin{array}{l}4 \\
8 \\
0\end{array}$ & $\begin{array}{l}0 . \\
3 \\
1 \\
5\end{array}$ & $\begin{array}{l}0 . \\
9 \\
0 \\
7 \\
\end{array}$ & $\begin{array}{l}0 . \\
12 \\
2\end{array}$ & $\begin{array}{l}0 . \\
65 \\
9\end{array}$ & $\begin{array}{l}0 . \\
20 \\
0\end{array}$ & $\begin{array}{l}0 . \\
07 \\
6\end{array}$ & $\begin{array}{l}0 . \\
30 \\
3\end{array}$ & $\begin{array}{l}0 . \\
04 \\
8\end{array}$ & $\begin{array}{l}0 . \\
35 \\
1\end{array}$ & $\begin{array}{l}0 . \\
07 \\
7\end{array}$ & $\begin{array}{l}0 . \\
23 \\
4\end{array}$ & $\begin{array}{l}1 \\
c \\
4\end{array}$ & c: & $\begin{array}{l}0 . \\
03 \\
3\end{array}$ & $\begin{array}{l}0 . \\
15 \\
0\end{array}$ & $\begin{array}{l}0 . \\
04 \\
3\end{array}$ & $\begin{array}{l}0 . \\
01 \\
3\end{array}$ & $\begin{array}{l}3 . \\
5 \\
7 \\
9 \\
\end{array}$ & $\begin{array}{l}1.0 \\
0\end{array}$ & $\begin{array}{l}0 . \\
90\end{array}$ & $\begin{array}{l}0.9 \\
6\end{array}$ \\
\hline $\begin{array}{l}\text { Mean } \\
\mathrm{Cl}\end{array}$ & $\begin{array}{l}7 . \\
7 \\
3\end{array}$ & $\begin{array}{l}0 . \\
29\end{array}$ & $\begin{array}{l}2 \\
4 \\
6 \\
\end{array}$ & $\begin{array}{l}0 . \\
2 \\
3 \\
5 \\
\end{array}$ & $\begin{array}{l}0 . \\
6 \\
0 \\
0\end{array}$ & $\begin{array}{l}0 . \\
09 \\
1\end{array}$ & $\begin{array}{l}0 . \\
46 \\
4\end{array}$ & $\begin{array}{l}0 . \\
15 \\
3\end{array}$ & $\begin{array}{l}0 . \\
05 \\
9\end{array}$ & $\begin{array}{l}0 . \\
20 \\
6\end{array}$ & $\begin{array}{l}0 . \\
03 \\
8\end{array}$ & $\begin{array}{l}0 . \\
25 \\
4\end{array}$ & $\begin{array}{l}0 . \\
05 \\
7\end{array}$ & 16 & $\begin{array}{l}0 . \\
72 \\
;\end{array}$ & $\begin{array}{l}0 . \\
16 \\
8\end{array}$ & $\begin{array}{l}0 . \\
02 \\
5\end{array}$ & $\begin{array}{l}0 . \\
10 \\
7\end{array}$ & $\begin{array}{l}0 . \\
02 \\
8\end{array}$ & $\begin{array}{l}0 . \\
00 \\
8\end{array}$ & $\begin{array}{l}2 . \\
5 \\
4 \\
1 \\
\end{array}$ & $\begin{array}{l}1.0 \\
0\end{array}$ & $\begin{array}{l}1 . \\
00\end{array}$ & $\begin{array}{l}1.0 \\
0\end{array}$ \\
\hline
\end{tabular}

${ }^{1} E M$ : El Médano; ${ }^{2} \mathrm{~A}$ : Asuka; $\Sigma R E E$ is sum of RE $\mathrm{E}$ irı $\mathrm{m}$ La to $\mathrm{Lu}$ in ppm. Normalized ratios are showing the ratio elements normalizer '? し'-chondrites. 


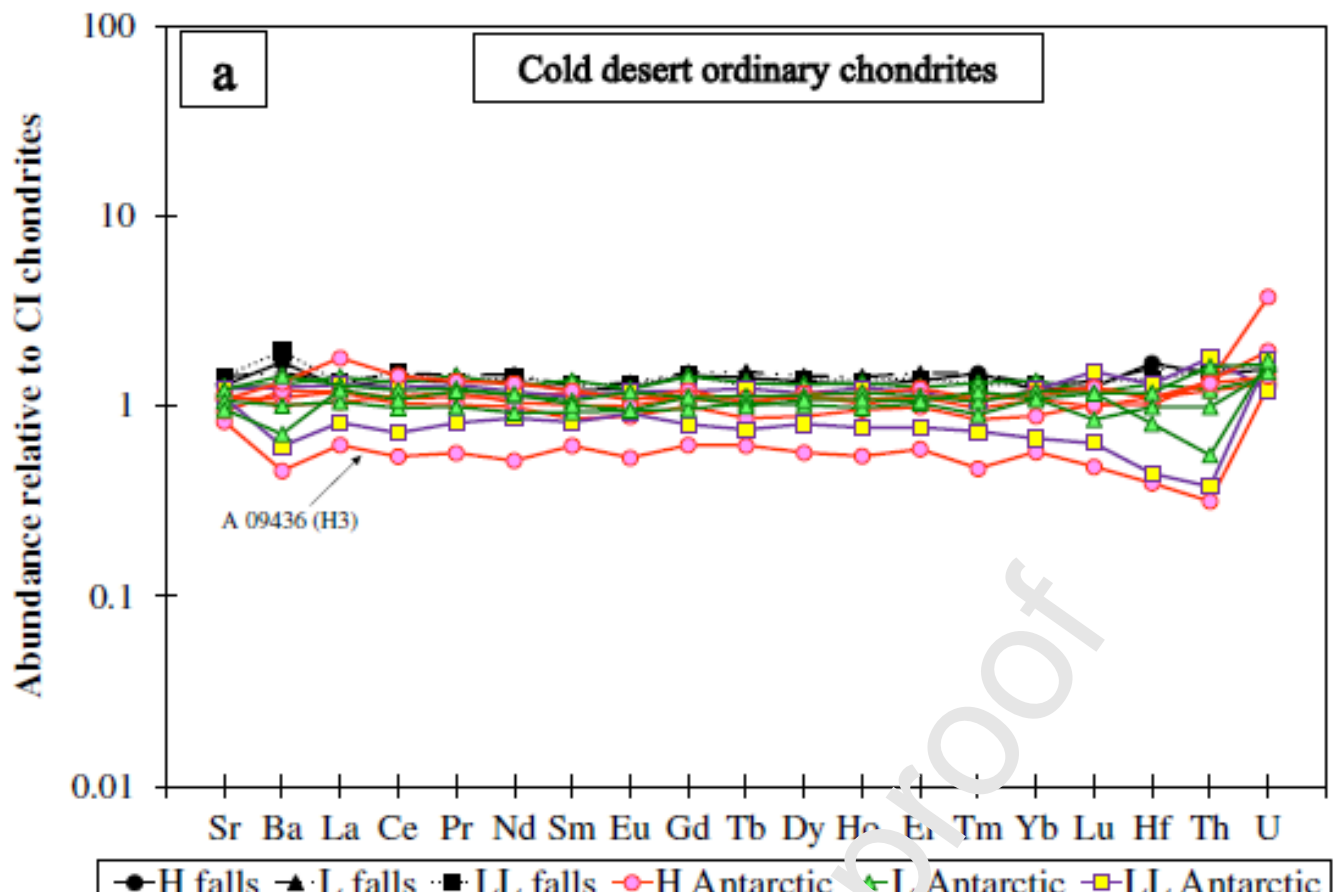

$\bullet-\mathrm{H}$ falls $\rightarrow$ L falls - LL falls $-\mathrm{O}-\mathrm{H}$ Antarctic $\leftarrow$ Antarctic - - -LL Antarctic

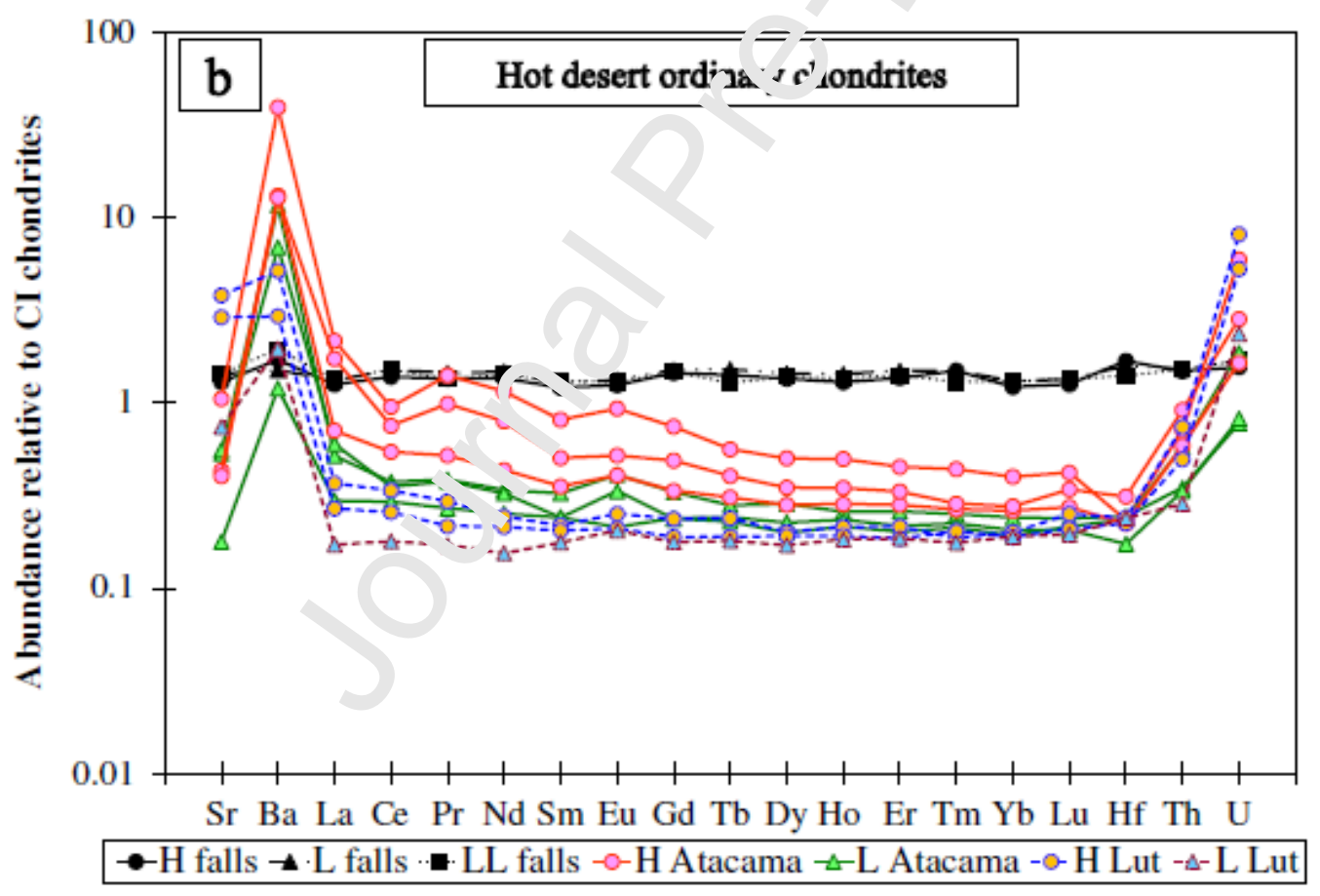

Fig. 1: Cl-normalized trace element composition of ordinary chondrites collected from a) Asuka dense collection area in Antarctic and b) Atacama and Lut hot deserts. Average $\mathrm{Cl}$ and ordinary chondrites abundances are from (Barrat et al., 2012) and (Wasson and Kallemeyn, 1988), respectively. 
Analyzed samples from Antarctica show relatively unfractionated REE patterns (average $\mathrm{La}_{N} / \mathrm{Lu}_{N}$ ratio is $1.15 \pm 0.18$ ) (Fig. 1a), almost mimicking those of the average fall compositions. However, most Antarctic samples show slightly lower REE concentrations than average falls. Average $\Sigma R E E$ for ordinary chondrites falls is 3.54 (Wasson and Kallemeyn, 1988), while our Antarctic samples' average $\Sigma$ REE is 2.71 $(\mathrm{n}=11)$. Strontium, $\mathrm{Ba}, \mathrm{Hf}$, and in some cases Th show very similar behavior to the REE in the Antarctic meteorites. These variations do not show a pronounced relative fractionation and as such mostly can be explained by differences in the equilibrium states of different chondrites, and the wide range in ordinary chondrite chemical compositions. For example, Asuka 09436 is a $\mathrm{H} 3$ chondrito and shows the lowest $\sum$ REE content (Fig 1a). The highly mobile $U$ contents ar ' sir vilar to the falls values, except Asuka 09618 with a high weathering degree ( :) t $t$ at shows an elevated $U$ concentration. Barium shows a slight depletion in Ariarctic samples with a generally lower $\mathrm{Ba} / \mathrm{La}$ ratio of these meteorites relative to $\mathrm{L} . n \mathrm{~s}$ of mean falls.

In comparison to the Antarctic meteorites int desert samples show larger disturbance and REE fractionation relativc to uie average fall values. $\Sigma R E E$ contents in the hot desert meteorites are lowe. th' $\mathrm{n}$ the average fall values, ranging from 1.09 to 2.42 in $\mathrm{H}$ chondrites from Atacána. For $\mathrm{L}$ chondrites, Atacama samples range from 0.65 to 0.87 and the averag $1 . r$ tnree Lut samples is 0.56 , both lower than the Atacama $\mathrm{H}$ chondrites. Maxim $\wedge$. , zıd average $(\mathrm{La} / \mathrm{Lu})_{N}$ ratio in the Atacama $\mathrm{H}$ chondrites is 5.15 and 4.27, espectively. Average ratios for Atacama $L$ and Lut (regardless of their types', ch( ndrites are 2.13 and 1.21 (respectively) and higher than the aforementioned $\mathrm{Ar} \operatorname{tarc}$ 'ic meteorites.

All the hot desert saı ples (except Kerman 009 and Kerman 013 from Lut, and EM 091 from Atacama) are depleted in $\mathrm{Sr}$, while in contrast, $\mathrm{Ba}$ is showing a remarkable uniform positive anomaly of greater than 1 . This anomaly reaches up to $\sim \times 39$ higher than average $\mathrm{Cl}$ chondrites in El Médano (EM) 091. Hafnium and Th contents in all hot desert samples are depleted compared to the average falls. However, the key difference is higher ratios of $\mathrm{Th} / \mathrm{Hf}$ and $(\mathrm{Lu} / \mathrm{Hf})_{N}$ in comparison to the falls. As shown in the case of EM 091, $(\mathrm{Lu} / \mathrm{Hf})_{N}$ can vary from 0.70 (close to mean fall values) to 1.71 in two different cuts from the same powder of a meteorite. Similar to Antarctic meteorites, hot desert samples display a positive $U$ anomaly, though with a somewhat greater magnitude. Except for two Atacama L chondrites (EM 89, 90), U concentrations in the analyzed hot desert samples are higher than the values 
reported for the average falls. The maximum measured content is for Kerman 009 which has $\sim \times 8$ times higher $\mathrm{U}$ than average $\mathrm{Cl}$ chondrites.

\subsection{Samarium-Nd systematics}

The results of the Sm-Nd isotopic measurements for the analyzed hot desert chondrites are reported in Table 4. As shown in Fig. $2,{ }^{147} \mathrm{Sm} /{ }^{144} \mathrm{Nd}$ ratios in the hot desert meteorites exhibit significant deviations from the chondritic isochron and the values reported for ordinary chondrite falls. The ${ }^{147} \mathrm{Sm} /{ }^{144} \mathrm{Nd}$ ratios measured in hot desert chondrites range from 0.0777 to 0.1973 , most often lower than the average of $0.1958 \pm 0.0012$ ( $n=22)$ reported for falls (Bouvier et al., ?noc: Patchett et al., 2004). The average ${ }^{147} \mathrm{Sm} /{ }^{144} \mathrm{Nd}$ for the Atacama and Lut samn $\mathrm{k}$, s $0.1433 \pm 0.0287(\mathrm{n}=10)$ and $0.1876 \pm 0.0080(n=7)$, respectively. This indice $\epsilon_{i}=$. higher variation and lower values of ${ }^{147} \mathrm{Sm} /{ }^{144} \mathrm{Nd}$ for the Atacama samples t' ia triose from the Lut Desert. The average ${ }^{147} \mathrm{Sm} /{ }^{144} \mathrm{Nd}$ ratios of the $\mathrm{H}$ and $\mathrm{L}$ chond'ite = from the Atacama are $0.1345 \pm$ $0.0287(n=7)$ and $0.1638 \pm 0.0147(n=3) . F(r '-L:$ Desert $H$ and $L$ chondrites the ratios are $0.1790 \pm 0.0037(n=3)$ and $0.1940 \_7 . \mathrm{i} 22(n=4)$, respectively. In both cases, H chondrites show higher deviations frorı, he falls than their $L$ counterparts.

Table 4: Sm-Nd isotopic comr osi:'ins of chondrite whole rocks. Elemental concentrations unit is $\mu \mathrm{g} / \mathrm{g}$

\begin{tabular}{|c|c|c|c|c|c|}
\hline Meteorite & $=n_{i}: i d$ & ${ }^{147} \mathrm{Sm} /{ }^{144} \mathrm{Nd}$ & ${ }^{143} \mathrm{Nd} /{ }^{144} \mathrm{Nd}$ & 2 se & $\varepsilon N d$ \\
\hline $\mathrm{CeC}^{1} 006$ & $J .2558$ & 0.1546 & 0.512555 & $5 E-06$ & -1.46 \\
\hline$E^{2} M^{2} 023$ & 0.2519 & 0.1523 & 0.512624 & $8 \mathrm{E}-06$ & -0.12 \\
\hline EM 049 & 0.2683 & 0.1622 & 0.512591 & $5 E-06$ & -0.76 \\
\hline EM 089 & 0.2722 & 0.1645 & 0.512567 & $8 \mathrm{E}-06$ & -1.23 \\
\hline EM 090 & 0.3051 & 0.1845 & 0.512559 & 7E-06 & -1.38 \\
\hline EM 091 & 0.1285 & 0.0777 & 0.512532 & $4 \mathrm{E}-06$ & -1.91 \\
\hline EM 091 duplicate & 0.2125 & 0.1285 & 0.512537 & 4E-06 & -1.82 \\
\hline EM 111 & 0.2632 & 0.1591 & 0.512579 & 4E-06 & -0.99 \\
\hline EM 120 & 0.1985 & 0.1200 & 0.512614 & $4 \mathrm{E}-06$ & -0.31 \\
\hline EM 120 duplicate & 0.2136 & 0.1292 & 0.512602 & 4E-06 & -0.55 \\
\hline Kerman 002 & 0.3172 & 0.1918 & 0.512593 & $2 \mathrm{E}-05$ & -0.73 \\
\hline Kerman 003 & 0.3180 & 0.1922 & 0.512563 & $8 \mathrm{E}-06$ & -1.30 \\
\hline Kerman 009 & 0.2884 & 0.1744 & 0.512517 & $6 E-06$ & -2.20 \\
\hline Kerman 013 & 0.3034 & 0.1834 & 0.512544 & 9E-06 & -1.68 \\
\hline Kerman 026 & 0.3222 & 0.1948 & 0.512695 & $2 \mathrm{E}-05$ & 1.28 \\
\hline
\end{tabular}




\begin{tabular}{|c|l|l|l|l|l|}
\hline Kerman 026 duplicate & 0.3263 & 0.1973 & 0.512713 & $2 \mathrm{E}-05$ & 1.61 \\
\hline Shahdad & 0.2966 & 0.1793 & 0.512575 & $2 \mathrm{E}-05$ & -1.07 \\
\hline
\end{tabular}

${ }^{1}$ Caleta el Cobre; ${ }^{2}$ El Médano

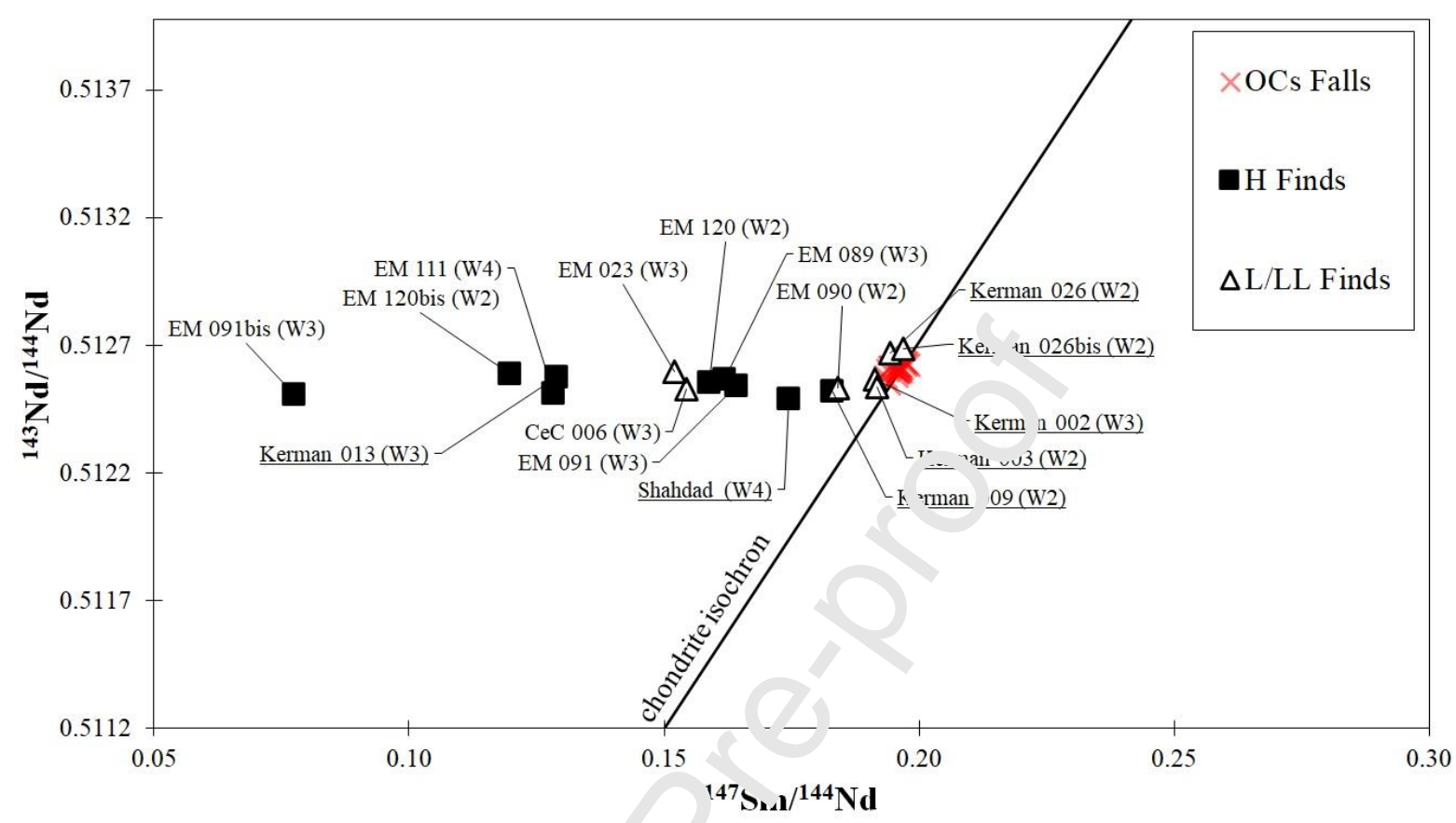

Fig. 2: Sm-Nd diagram for whole-rock $m_{1}$ teorites collected from Atacama and Lut hot deserts. El Médano (EM) and Cal $+\neg c^{\prime}$ Cobre $(\mathrm{CeC})$ are from the Atacama desert. Kerman and Shahdad are from the I ut desert (underlined). Data for ordinary chondrites falls are from (Bo ivir et al., 2008). Standard deviation of OCs falls values is $0.0012(n=22)$.

In addition to varia:ir, between different meteorites, differences in ${ }^{147} \mathrm{Sm} /{ }^{144} \mathrm{Nd}$ also occur within a given meteorite as evidenced by different values measured for duplicates which have been taken from the same powder. For instance, the standard deviation between two ${ }^{147} \mathrm{Sm} /{ }^{144} \mathrm{Nd}$ measurements of Kerman 026 is 0.0013 , while for EM 091 and EM 120 it is $0.0254(n=2)$ and $0.0046(n=2)$, respectively. It should be noted that Kerman 026 was found in the Lut Desert, while the two latter are from the Atacama Desert. Note that EM 091, the sample with the highest standard deviation, is visually more weathered than the other two samples.

The measured ${ }^{143} \mathrm{Nd} /{ }^{144} \mathrm{Nd}$ ratios are expressed as $\varepsilon \mathrm{Nd}$ values, where $\varepsilon \mathrm{Nd}$ is calculated using the equation shown below: 
$\left.\varepsilon \mathrm{Nd}=\left[\left({ }^{143} \mathrm{Nd} /{ }^{144} \mathrm{Nd}\right)\right)_{\text {sample }} /\left({ }^{143} \mathrm{Nd} /{ }^{144} \mathrm{Nd}\right)_{\mathrm{CHUR}}\right]-1 \times 10^{4}$

where CHUR is the chondritic uniform reservoir with a composition of ${ }^{143} \mathrm{Nd} /{ }^{144} \mathrm{Nd}=$ 0.51263 (Bouvier et al., 2008). Figure 3 depicts the $>4 \varepsilon N d$-unit variation measured for desert meteorites The $\varepsilon N d$ values range from -2.20 to 1.61 , which is greater than the -1.07 to 0.64 range for falls (Bouvier et al., 2008). The average $\varepsilon N d$ value for samples from the Atacama and Lut are -1.05 and -0.58 , respectively.

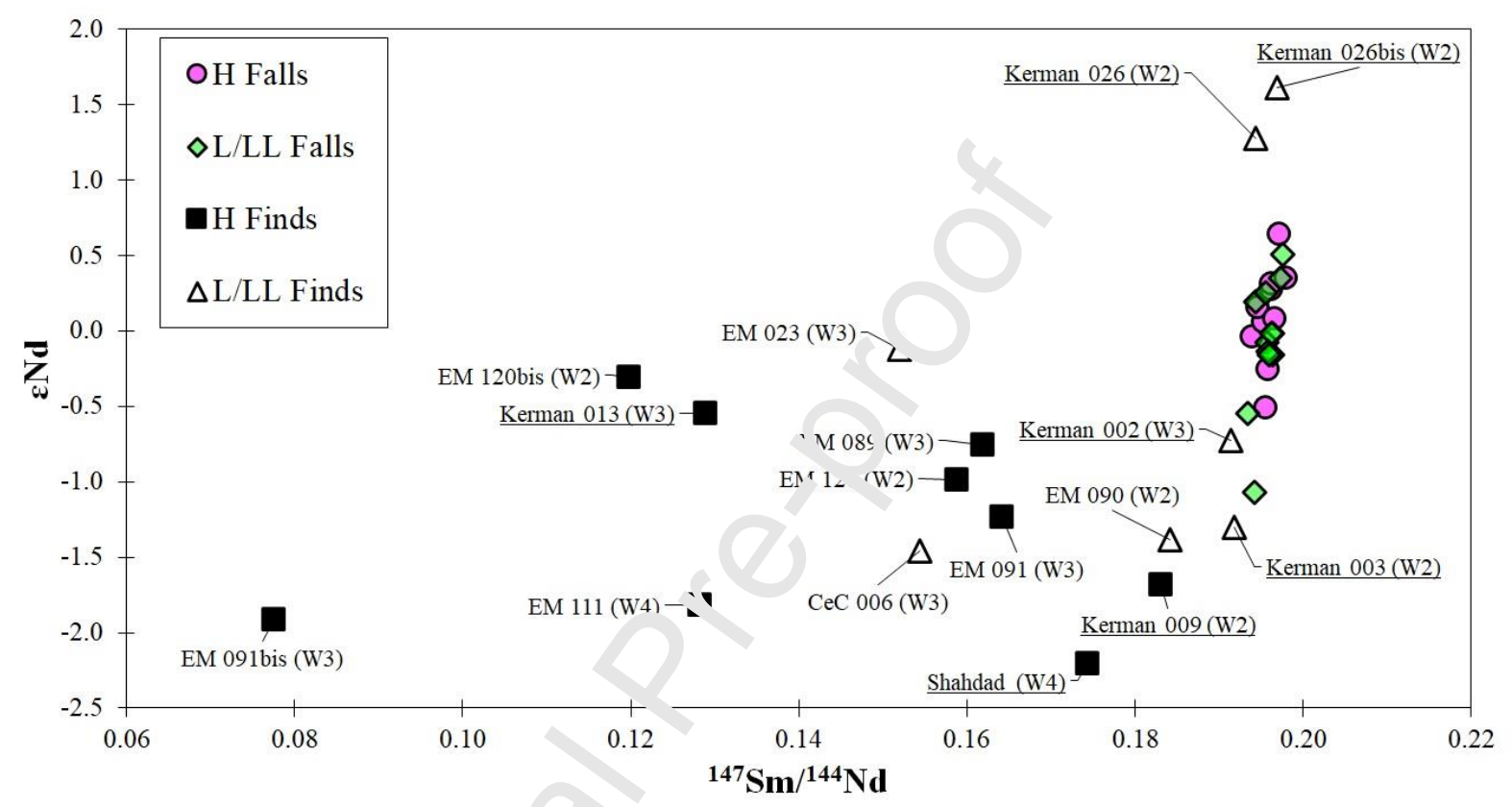

Fig. 3: ${ }^{147} \mathrm{Sm}^{/ 144} \mathrm{Nd}$ versus $\varepsilon \mathrm{Nc}$ oi the analyzed hot deserts compared to the fall ordinary chondrites. El Méda 70 (EM) and Caleta el Cobre (CeC) are from the Atacama Desert. Kermar, anc Shahdad are from the Lut Desert (underlined). Data for fall ordinary chondrites are from (Bouvier et al., 2008).

\section{DISCUSSION}

Our data confirms that terrestrial weathering of meteorites changes their trace element ( $\mathrm{Sr}, \mathrm{Ba}, \mathrm{REE}, \mathrm{Hf}$, Th, and $\mathrm{U}$ ) concentrations. In addition, $\mathrm{Sm}-\mathrm{Nd}$ isotopic measurements of ordinary chondrites from the Atacama and Lut hot deserts show significant effects of terrestrial weathering as manifested by their non-CHUR ${ }^{147} \mathrm{Sm} /{ }^{144} \mathrm{Nd}$ and ${ }^{143} \mathrm{Nd} /{ }^{144} \mathrm{Nd}$ ratios.

\subsection{A comparison of the trace element compositions of chondrites from hot and cold deserts}


Thousands of years of exposure to a terrestrial environment leads to chemical contamination of meteorites (Al-Kathiri et al., 2005; Hezel et al., 2011; Pourkhorsandi et al., 2017a). Interaction with liquid water containing different elements originating from the soil below the meteorite and the atmosphere, and chemical leaching by these liquids, plus physical and biological weathering are common in hot desert environments. All these weathering processes lead to the modified chemical patterns (Fig. 1b). Also evident in our data, are anomalies in Sr, Ba, REE, Hf, Th and $\mathrm{U}$ contents, which are well-known indicators of hot desert weathering (Al-Kathiri et al., 2005; Barrat et al., 2003; Folco et al., 2007; Hezel et al., 2011; Hofmann et al., 2018; Moore and Brown, 1963; Pourkhorsandi et al., 2017a; SaL..'or et al., 2010; Stelzner et al., 1999; Zeng et al., 2018; Zurfluh et al., 2012). In c( mp. rison, Antarctic meteorites show less chemical modification than thos $\ominus$ frı $m$ the hot deserts (Fig. 1).

Starting with $\mathrm{Sr}$ and $\mathrm{Ba}$, our data shows the chelı 'ical differences between different hot deserts in addition to differences to the Antarct心 samples. Meteorites from different find locations have different trends $\cap B$ z and Sr contents (Fig. 4). Relative to

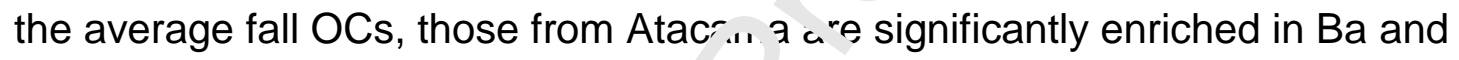
depleted in $\mathrm{Sr}$, while two Lut samples a a highly enriched in $\mathrm{Sr}$. In comparison, Antarctic samples show values sligr.:'v lower than the average fall values. These differences can be explained by $t .7$; c umpositional differences of the meteorite residence sites and different s il cumpositions. For instance, in the Lut Desert, terrestrial fluids containing $\mathrm{Sr}$ : hat originated from carbonate-rich soils infiltrate the meteorites through cracks, ' ' ' ich ultimately leads to an increase in the Sr budget of the rock. This scena ${ }^{-i o}$ : c r,onsistent with higher carbonate contents in Lut Desert meteorites than those fr $\mathrm{Jm}$ other hot deserts as reported in (Pourkhorsandi et al., 2019). Similar to Lut meteorites, high $\mathrm{Sr}$ contents in samples recovered from carbonate-rich soils of deserts in Oman are also in accordance with such a scenario (Al-Khatiri et al., 2005). Gibson and Bogard (1978) reported the occurrence of such uptake of Sr within as few as 19 years after meteorite fall. Enriched Ba patterns in the Atacama meteorites can be explained by higher values of $\mathrm{Ba}$ in these soils (360-954 ppm) reported by (Pourkhorsandi et al., 2017a) compared to the soil samples collected from the Lut Desert (332-562 ppm) reported by (Pourkhorsandi et al., 2019). In some cases, possible small-scale soil compositional heterogeneities might result in weathered meteorites with relatively different chemical compositions than the 
majority of recovered meteorites from that region. For instance, this might be the case for Kerman 026, which regardless of being from the Lut Desert, has lower Sr values than mean falls. Contrary to the hot desert meteorites, the meteorite finds from Asuka DCA have not been in contact with soil. Our analyzed samples from the Asuka were residing on the ice at the time of their recovery. Relative to hot desert meteorites, terrestrial weathering has not been sufficiently intense to alter their chemical compositions significantly. The active weathering processes in such an environment would be abrasion by the wind and leaching of the meteorite during its limited interaction with liquid water below and on the ice surface (Harvey, 2003;

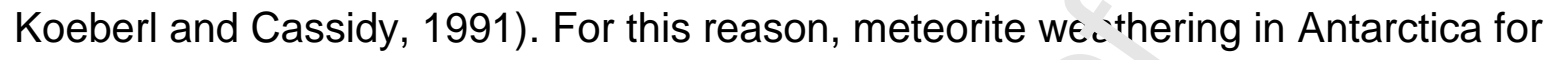
the majority of the elements would cause depletions ratl er $t$ lan enrichments (for instance in $\mathrm{Ba}, \mathrm{Hf}, \mathrm{Th}$, and some cases in REE as $s \in \geqslant n$ I, Fig. 1a). Oxidation of chondritic components such as troilite by liquid wate, forms acidic environment which itself facilitates alteration (leaching) process of ou or minerals such as Ca-phosphate which is the main host for these elements in a neteorite (Ebihara and Honda, 1983; Jones et al., 2014; Martin et al., 2013). Hc vever, there are some exceptions such as $U$ which can be enriched compared $t \cdot$ f $f$. Is. Uranium enrichment in both falls, and Antarctic finds has been also repc. ${ }^{+}$ed from meteorites collected from other regions of the continent, with the effects of $I \mathcal{L}_{2}^{\prime}$ ' sources, such as water sprays from the Southern Ocean, or local rock $2 \lambda_{r} \cap 0$ sures proposed as being responsible (e.g., Harvey, 2003). 


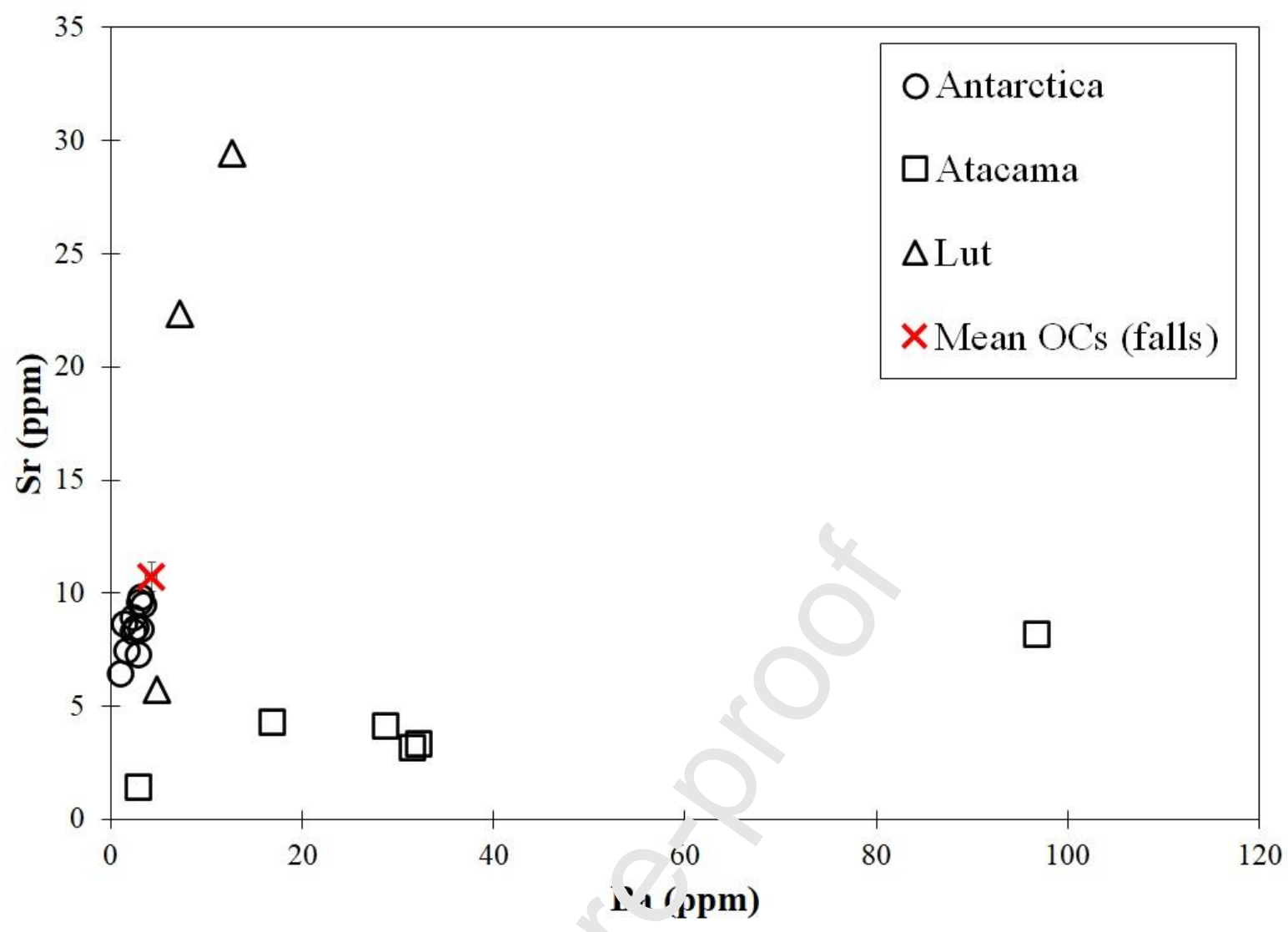

Fig. 4: Absolute values of Ba versus S, in the Atacama and Lut deserts, and Antarctica samples. Average ordinai;' chondrite values from (Wasson and Kallemeyn, 1988) are plotted for $\mathrm{v}$ m marison. Error bars show standard deviation of average ordinary chondrites.

Terrestrial weathering has fecreased the REE contents in hot desert meteorites and probably to some rey 'ee in Antarctic meteorites. However, it has not significantly affected the ratios of 1 ise elements in Antarctic samples, suggesting possibly insignificant re-adjustment of $\mathrm{Sm}-\mathrm{Nd}$ isotopic system. This makes the majority of samples (or at least ordinary chondrites) collected from Asuka reliable for cosmochemical studies. Relatively significant weathering effects occur in highly altered meteorites such as in Asuka 09436, which has a weathering degree $C$ and shows the lowest REE content. However, in the other samples the chemical composition does not show any relationship with weathering degree. Roughly $61 \%$ of Antarctic basaltic achondrites (especially eucrites) exhibit a cerium anomaly (Kagi and Takahashi, 1998; Mittlefehldt and Lindstrom, 1991), and it has been suggested to be a fingerprint of chemical removal of REE from these meteorites. However, we 
do not observe it in our samples, which suggests it is either limited to achondrites or it points to the presence of multiple weathering regimes in the Antarctica.

Unlike in the Antarctic finds, hot desert weathering appears to modify both REE abundances and ratios, likely due to concomitant action of two main processes. One is contamination by terrestrial fluids coming from soil resulting in increased light$\mathrm{REE} /$ heavy-REE ratios (represented by higher $\mathrm{La}_{N} / \mathrm{Lu}_{N}$ ratios), and second leaching of chondritic components such as Ca-phosphates causing a decrease in bulk REE budget (Table 3) (Al-Kathiri et al., 2005; Pourkhorsandi et al., 2017a). As suggested by (Pourkhorsandi et al., 2017a), the longer average terrestrial residence times of the Atacama meteorites are responsible for their more fractionate $\lambda$ REE patterns in comparison to those from the Lut Desert. However, their : igner abundances of $\Sigma R E E$ can be explained by the lower weathering rate and nc* imited leaching than those from the Lut Desert (Pourkhorsandi et al., 2019). (Di zrıd et al., 2006) suggest a faster weathering rate for $\mathrm{H}$ than $\mathrm{L}$ and $\mathrm{LL} \mathrm{OCs}$, due to thir higher (Fe,Ni) metal contents leading to a higher abundance of Fe oxy-hy Ir sx des which can absorb REE from the fluids and soil and hence show high R ${ }_{-L}-\mathrm{C}$ ntamination at the microscale (Crozaz et al., 2003; Crozaz and Wadhwa, 2001). 'tigher REE fractionation in these meteorites can be explained by this process. h' 'wever, comparison of the weathering degrees with REE composition shows a cur Ip Iex pattern. The meteorite EM 120 with a weathering degree W2 shows the second highest fractionation $(L a N / L u N=5.07)$ after EM 091 (H5 W3, LaN/LuN - -15), while the strongly weathered (W4) EM 111 is the least fractionated $\mathrm{H}$ chona ${ }_{1}$ :c from the Atacama.

Similar to REE, $\mathrm{Ht}$, na Th have been affected by terrestrial weathering. This is the case for some Asuka samples and for all hot desert meteorites. As mentioned earlier, these two elements partly are fixed in Ca-phosphate structure and can get released by alteration of this mineral during weathering. Thorium is not only found in Caphosphates as Kuznetsov (2011) reported $5 \%$ of thorium in water-soluble components and $9.1 \%$ in troilite, both of which potentially can be easily affected by weathering. Uranium enrichment is the other weathering proxy that has been observed in hot desert meteorites which is suggested to form as a result of contamination by soil fragments and interaction with fluids originating from the soil (e.g., Hezel et al., 2011). As a consequence of these processes, Lu/Hf and Th/U ratios in our analyzed meteorites, in particular in hot desert samples have been 
modified. Main results of such modification would be disturbances in Lu-Hf and U-Th$\mathrm{Pb}$ isotopic systems which also have been reported by Bast et al. (2017) and Weber et al. (2017).

In conclusion, we see that the Antarctic meteorites are chemically less affected by terrestrial weathering than those from the hot deserts. Soil composition controls $\mathrm{Sr}$ and $\mathrm{Ba}$ contents of hot desert meteorites. Rare Earth element ratios in Antarctic samples are close to the average falls. By contrast, in the hot desert samples REE are strongly affected by weathering, as evidenced by both lower absolute values and higher relative fractionations. The maximum fractionation is observed in the $\mathrm{H}$ chondrites from the Atacama. Meteorite weathering degrans 'o not show straightforward relationships with their REE compositinns. L'igher contamination of

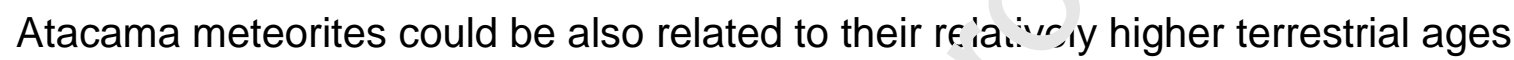
than those from other regions (Drouard et al., 20,y). I ogether, this evidence makes hot desert meteorites, and particularly those frori $t ı, ?$ Atacama, suspect samples for cosmochemical studies, in contrast to the qi extt $r$ reliability of Antarctic meteorites for such studies.

\subsection{Modification of $\mathrm{Sm}-\mathrm{Nd}$ isntopic zystematics during terrestrial weathering}

The previous section showed the $\dagger_{\lrcorner} \cdot{ }^{-}$strial weathering can fractionate elemental parent/daughter ratios, and the reire suggests that special care must be taken into account when working with $\mathrm{r}$. h-Sr, U-Th- $\mathrm{Pb}$, and even Lu-Hf isotopic systems particularly in hot desert inete urites. Such fractionation will create deviations from "true" isochrons. St' 'di 's u ilizing Rb-Sr, U-Pb, or Lu-Hf chronometers such as (Bast et al., 2017; Borg et al., 2016, 2003; Debaille et al., 2007; Dreibus et al., 2001; Elardo et al., 2014; Shih et al., 2007; Sokol et al., 2008; Tatsumoto et al., 1981) have all reported such cases. The disruptive effects of terrestrial weathering on $\mathrm{Rb}-\mathrm{Sr}$, $\mathrm{Lu}-\mathrm{Hf}$, and $\mathrm{U}-\mathrm{Th}-\mathrm{Pb}$ isotopic system are to be expected from the $\mathrm{Sr}$ and $\mathrm{U}$ positive anomalies and modified $\mathrm{Lu} / \mathrm{Hf}$ and $\mathrm{Th} / \mathrm{U}$ ratios (Fig. 1). However, in the case of Sm$\mathrm{Nd}$ isotopic system, it is complicated as little is known about their respective behaviors during terrestrial weathering.

In addition to our data regarding modification of elemental abundances of REE, isotopic measurements can offer supplementary evidence of modification of $\mathrm{Sm}-\mathrm{Nd}$ isotopes during terrestrial weathering. This is a crucial issue as hot desert meteorites, 
in particular Saharan achondrites, are amongst the main objects used for cosmochemical studies.

In their Sm-Nd study on the Golpara ureilite, Torigoye-Kita et al. (1995) suggested that terrestrial contamination, mainly concentrated in the acid leachates, was responsible for its anomalous composition. Jagoutz et al., (2001) suggested a large shift in the $\mathrm{Sm} / \mathrm{Nd}$ ratio caused by contamination to be responsible for a hot desert martian meteorite's "anomalous" isotopic composition. In addition, several other works such as Debaille et al. (2007), Dreibus et al. (2001), and Elardo et al. (2014) have yielded data that partly correlated with these findings. Outside of these studies, the majority of works on hot desert meteorites using Sm-N// L गtopic system suggest very low probability of disturbance due to terrestrial weat, $\_$n.ng (Debaille et al., 2007).

Most of the data available on modifications of $\mathrm{St}_{1}$. $\mathrm{Na}$ isotopic system during weathering deal with achondrites. However, as $\because$ eat ering mainly involves interaction of fluids with primary components thorough cracks, tris would apply to ordinary chondrites as well. In fact, higher abundar $i \epsilon \frown f(\mathrm{Fe}, \mathrm{Ni})$ metal and troilite in ordinary chondrites would offer a higher altere .or capacity and higher chemical modification for ordinary chondrites.

As shown in the previous sect or i $^{-}$Table 4 and Fig. $5, \mathrm{Sm} / \mathrm{Nd}$ ratios in our analyzed hot desert samples $=$ re i iver than the average falls. Disturbance of this ratio is responsible for lower ${ }^{147} \mathrm{Sm} /{ }^{144} \mathrm{Nd}$ ratio which is more evident in the Atacama samples as they have mu: $\because \mathrm{fl}$ actionated REE patterns. Lower $\mathrm{Sm} / \mathrm{Nd}$ ratios show the effects of mixing with a ter estrial component (e.g., Torigoye-Kita et al., 1995). The majority of Atacama $m \in$ eorites regardless of their weathering degrees (at least $W \geq 2$ ) are indeed contaminated. 


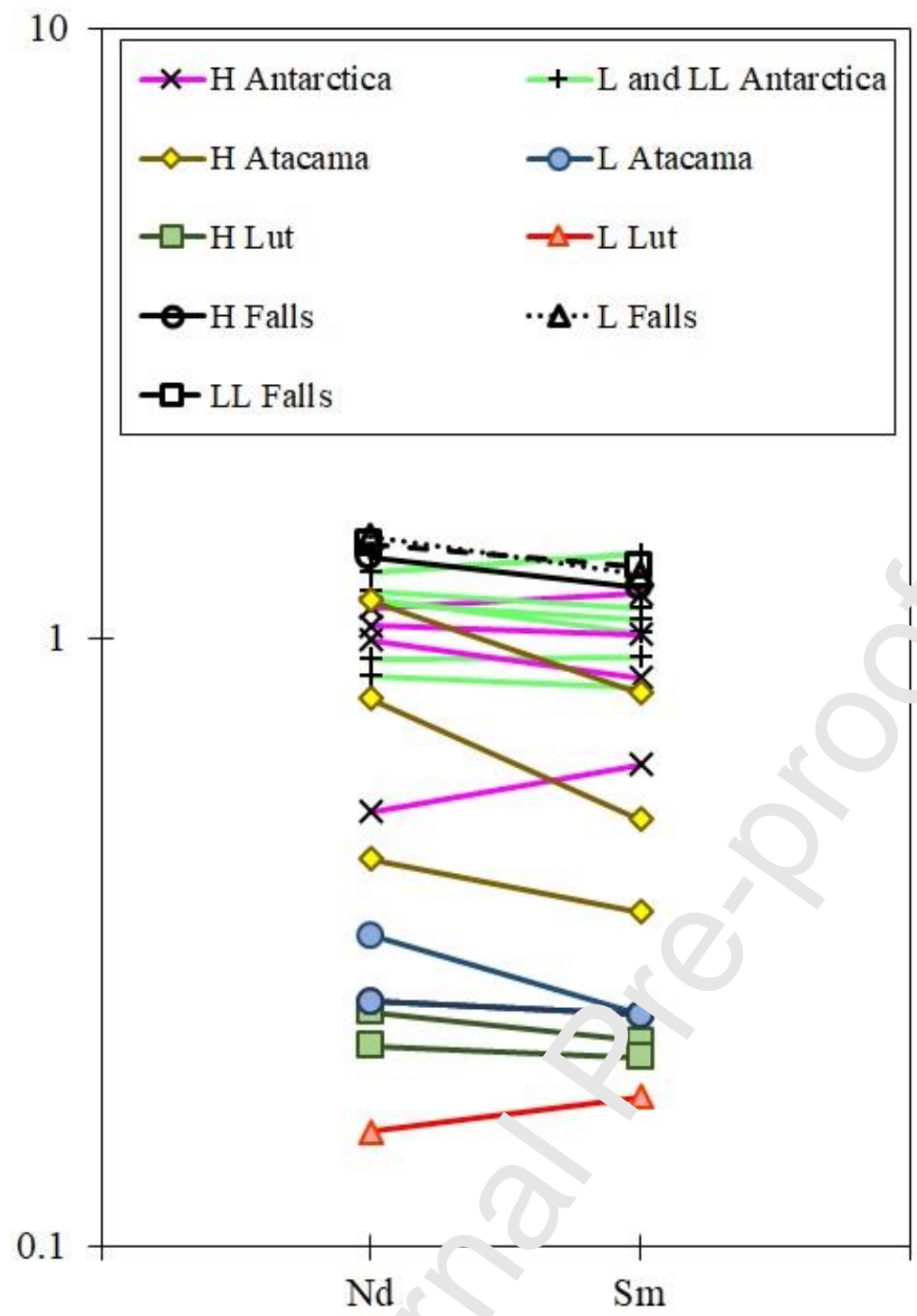

Fig. 5: Cl-normalized abur. va nces of Sm and Nd in whole-rock Antarctic, Atacama Desert, and Lut De on ord nary chondrites. Average ordinary chondrite and $\mathrm{Cl}$ chondrite values from ( Vasson and Kallemeyn, 1988) and (Barrat et al., 2012).

Comparison of the ${ }^{147} \mathrm{Sm} /{ }^{144} \mathrm{Nd}$ ratio with meteorite types shows higher deviations from the average value fall for $\mathrm{H}$ chondrites (Fig. 5). As in the case of elemental abundances, the same can be applied for isotopic compositions. The ${ }^{147} \mathrm{Sm} /{ }^{144} \mathrm{Nd}$ ratio does not show a straightforward relationship with weathering degree. It gets even more complicated as we see highly heterogeneous duplicates for two single meteorites (El Médano 091 and 120), while we do not observe this in two duplicates of Kerman 026 collected from the Lut Desert. 
As mentioned earlier, $\mathrm{La}_{N} / \mathrm{Lu}_{N}$ ratio is shown to increase during terrestrial weathering of meteorites. Plotting this ratio together with ${ }^{147} \mathrm{Sm} /{ }^{144} \mathrm{Nd}$ of relatively highly modified $\mathrm{H}$ chondrites (Fig. 6) shows a negative correlation with ${ }^{147} \mathrm{Sm} /{ }^{144} \mathrm{Nd}$ ratio. This correlation shows that both of these ratios are being controlled by similar processes, mainly mixing with terrestrial components. As REE elemental fractionation in meteorites proceeds towards a soil like composition during desert weathering, the ${ }^{147} \mathrm{Sm} /{ }^{144} \mathrm{Nd}$ ratios follow similar route towards terrestrial compositions.

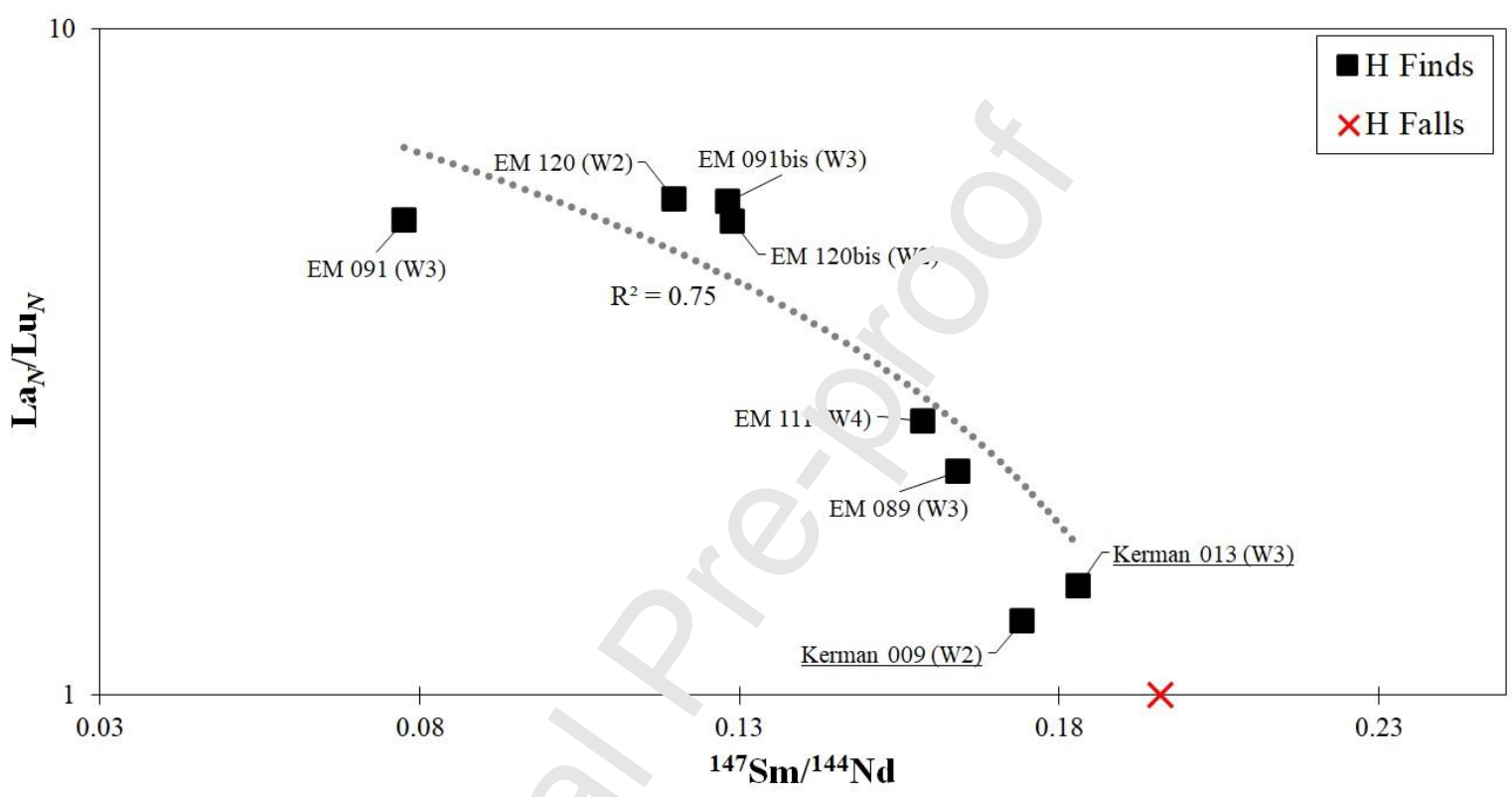

Fig. 6: ${ }^{147} \mathrm{Sm}^{1 / 144} \mathrm{Nd}$ versus $\mathrm{La}_{1}, \mathrm{Lu}_{N}$ of the analyzed hot deserts compared to the fall ordinary chondrites. Samp'? s 'rom Lut are underlined. Dashed curve shows linear trend pattern of $\mathrm{H}$ finds. Sicn'lard deviation for ${ }^{147} \mathrm{Sm}^{/ 144} \mathrm{Nd}$ of $\mathrm{H}$ falls in 0.0012 $(n=10)$. Average elt. $\eta \epsilon . ?$ ta' and isotopic ordinary chondrite values are from (Wasson and Kallemeyn, 1988! =nd (Bouvier et al., 2008), respectively.

A comparison between Ba concentrations, shown to get enriched during weathering, with ${ }^{147} \mathrm{Sm} /{ }^{144} \mathrm{Nd}$ ratios, reveals a negative correlation. As shown in Fig. 7, $\mathrm{H}$ chondrites with higher $\mathrm{Ba} / \mathrm{Nb}$ values (higher chemical alteration), have lower ${ }^{147} \mathrm{Sm} /{ }^{144} \mathrm{Nd}$ and show higher deviations from the CHUR value. As mentioned in the previous sections, Ba contents in these meteorites are higher in the samples collected from Ba-rich soils (Atacama) than those from the Lut Desert. 


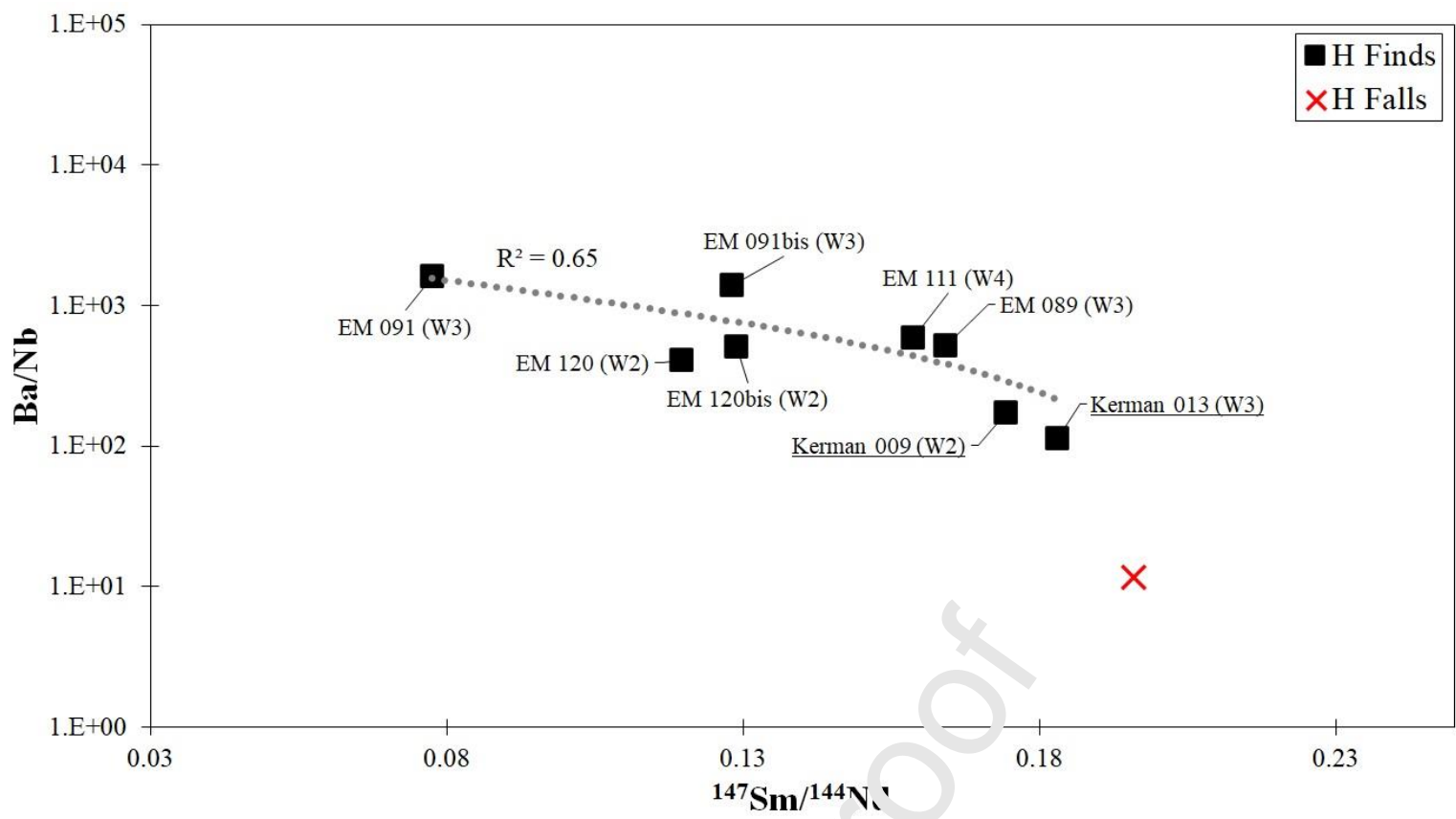

Fig. 7: ${ }^{147} \mathrm{Sm}^{1 / 44} \mathrm{Nd}$ versus $\mathrm{Ba} / \mathrm{Nb}$ of the analyze $\mathrm{ho}$. deserts compared to the fall ordinary chondrites. Samples from Lut are ur.d?rlined. Dashed curve shows linear trend pattern of $\mathrm{H}$ finds. Standard deviatic $1 \mathrm{tu}{ }{ }^{4+7} \mathrm{Sm}^{1 / 44} \mathrm{Nd}$ of $\mathrm{H}$ falls in 0.0012 $(n=10)$. Average elemental and isoto', ic ',rdinary chondrite values are from (Wasson and Kallemeyn, 1988) and (Bouvicr et al., 2008), respectively.

In addition to the $\mathrm{Sm} / \mathrm{Nd} \mathrm{znci}^{147} \mathrm{Sm} /{ }^{144} \mathrm{Nd}$ ratios, ${ }^{143} \mathrm{Nd} /{ }^{144} \mathrm{Nd}$ ratio is also highly disturbed (Fig.3). Except Kt.man 026, which as discussed earlier also shows

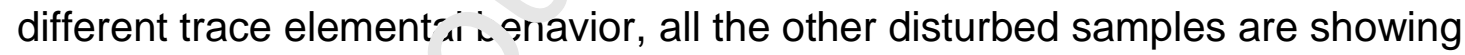
negative $\varepsilon N d$ values. Siln wilar to the ${ }^{147} \mathrm{Sm} /{ }^{144} \mathrm{Nd}$ ratio, $\varepsilon N d$ values do not show a straightforward relationship with the weathering degrees. However, as in the case of the previous discussions, the samples with the highest negative $\varepsilon N d$ values are $\mathrm{H}$ chondrites from the Atacama and Lut deserts.

More studies such as laser ablation ICP-MS in-situ measurements of trace elements will be needed to pinpoint the main factors controlling contamination of trace element compositions and $\mathrm{Sm}-\mathrm{Nd}$ isotopic composition of hot desert meteorites. Such studies should target different primary components, weathering cracks and chemical modifications around them, and possible terrestrial components inside weathered meteorites. Our data clearly shows that care must be taken into account while dealing with samples collected from hot deserts, even the fresh-looking 
ones, and including the Atacama Desert which is shown to be an extraordinary region hosting a high population of meteorites (Gattacceca et al., 2011), and in particular unique samples (e.g., Pourkhorsandi et al., 2017b).

\section{CONCLUSIONS}

Terrestrial weathering modifies trace element composition of ordinary chondrites, in particular REE. The degree of chemical modification in the Antarctic meteorites collected from the Asuka DCA is lower than that for hot desert meteorites. Antarctic meteorites show REE patterns close or only slightly below $t_{1}$. at of average falls, but without any noticeable fractionation. This also applies m. $s \mathrm{stl}$, for $\mathrm{Sr}, \mathrm{Hf}$, and $\mathrm{Th}$. Uranium contents in Antarctic samples are close to $f_{a}^{\prime}$ 'ls I alue with some enrichments possibly caused by weathering. Barium concentr $-:-2 \eta_{1}$ in Antarctic samples is lower than falls. Meteorite leaching (especially Ca-phosp ates) can explain this depletion. Hot desert meteorites show $\mathrm{Ba}$, LREE, and 'J 'unsitive anomalies which are representative of terrestrial weathering $C_{\mathcal{L}}$ ncentrations of REE in the hot desert meteorites is lower than average falls $r$ r.d also they are highly fractionated. This fractionation is higher in the Atacaı: 7 samples, especially $\mathrm{H}$ chondrites, than those from the Lut Desert. This is in ag e $\Leftrightarrow$, ant with their average residence on Earth: 700 kyr for Atacama meteorites ve sus $12 \mathrm{kyr}$ for other hot desert meteorites (Drouard et al., 2019). We suggest this $r_{1}$. nditication is the result of concomitant action of two main processes. One is cinte mination by terrestrial fluids coming from soil resulting in increased light- $R E E$, hea sy-REE ratios (represented by higher $L a_{N} / L u_{N}$ ratios), and second leaching of chol dritic components such as Ca-phosphates causing a decrease in bulk REE budget. Terrestrial weathering lowers $\mathrm{Sm} / \mathrm{Nd}$ ratios which in consequence disturbs ${ }^{147} \mathrm{Sm} /{ }^{144} \mathrm{Nd}$ composition. This ratio has a negative correlation with $\mathrm{La}_{N} / \mathrm{Lu}_{N}$ ratio. Barium content of the weathered desert meteorites show a negative correlation with ${ }^{147} \mathrm{Sm} /{ }^{144} \mathrm{Nd}$ contents. This makes $\mathrm{La}_{N} / \mathrm{Lu}_{N}$ ratio and $\mathrm{Ba}$ concentration proxies to check possible modification of ${ }^{147} \mathrm{Sm} /{ }^{144} \mathrm{Nd}$ ratio. Hot desert weathering changes ${ }^{143} \mathrm{Nd} /{ }^{144} \mathrm{Nd}$ composition of the meteorites as well, which is more evident in the $\mathrm{H}$ chondrites. This systematic work shows disturbance of $\mathrm{Sm}-\mathrm{Nd}$ isotopic composition of meteorites during terrestrial weathering. Disturbance of $\mathrm{Lu} / \mathrm{Hf}$ and $\mathrm{Th} / \mathrm{U}$ ratio also point to possible changes in $\mathrm{Lu}-\mathrm{Hf}$ and $\mathrm{U}-\mathrm{Th}-\mathrm{Pb}$ isotopic systems of these meteorites. Great caution should be paid when dealing with hot desert 
meteorites, even when they look fresh. More studies, especially in-situ measurements, should be conducted to pinpoint the main factors controlling REE and $\mathrm{Sm}-\mathrm{Nd}$ isotopic compositions during terrestrial weathering of meteorites.

\section{Declaration of competing interest}

The authors declare than they have no known competing interests or personal relationships than could have appeared to influence the work reported in this paper.

\section{Acknowledgments}

H. Pourkhorsandi, V. Debaille, P. Rochette, and J. Sntlacceca thank the PHC Tournesol program. H. Pourkhorsandi, R. M. G. Arniylaye, and V. Debaille acknowledge funding from the ERC StG "ISoSyr", a nd V. Debaille also thanks the FRS-FNRS for present support. G. Hublet and F.. Mkieda are thanked for insightful discussions. We thank W. Debouge and $S 、 x u$;hies for keeping the clean labs in perfect conditions. J. de Jong is thank za for naintaining the analytical instruments. We thank RBINS and CEREGE for prov ring meteorite samples from Antarctic and hot deserts, respectively. This proieci has received funding from the European Union's Horizon 2020 research an $1 \mathrm{i}$ inovation programme under the Marie Skłodowska-Curie grant agre€. nent No 801505.

\section{Declaration of interests}

The authors declare that th. $y$ have no known competing financial interests or personal relationships that could have appearea $\mathrm{u}$ influence the work reported in this paper.

The authors declare the following financial interests/personal relationships which may be considered as potential competing interests:

\section{References}

Al-Kathiri, A., Hofmann, B.A., Jull, A.J.T., Gnos, E., 2005. Weathering of meteorites from Oman : Correlation of chemical and mineralogical weathering proxies with $14 \mathrm{C}$ terrestrial ages and the influence of soil chemistry. Meteorit. Planet. Sci. 40, 1215-1239. https://doi.org/10.1111/j.1945-5100.2005.tb00185.x

Armytage, R.M.G., Debaille, V., Brandon, A.D., Agee, C.B., 2018. A complex history 
of silicate differentiation of Mars from $\mathrm{Nd}$ and $\mathrm{Hf}$ isotopes in crustal breccia NWA 7034. Earth Planet. Sci. Lett. 502, 274-283.

https://doi.org/10.1016/j.epsl.2018.08.013

Barrat, J.A., Jambon, A., Bohn, M., Blichert-Toft, J., Sautter, V., Göpel, C., Gillet, P., Boudouma, O., Keller, F., 2003. Petrology and geochemistry of the unbrecciated achondrite Northwest Africa 1240 (NWA 1240): An HED parent body impact melt. Geochim. Cosmochim. Acta 67, 3959-3970. https://doi.org/10.1016/S00167037(03)00092-9

Barrat, J.A., Zanda, B., Moynier, F., Bollinger, C., Liorzou, `., Bayon, G., 2012. Geochemistry of $\mathrm{Cl}$ chondrites: Major and trace ele' neı. 's, and $\mathrm{Cu}$ and $\mathrm{Zn}$ Isotopes. Geochim. Cosmochim. Acta 83, 79-92 https://doi.org/10.1016/j.gca.2011.12.011

Bast, R., Scherer, E.E., Bischoff, A. 2017. The ${ }^{1 / 2} \cdot . \cdot{ }^{176} \mathrm{Hf}$ systematics of ALM-A: A sample of the recent Almahata Sitta me ie'rite fall. Geochem. Perspect. Lett. 3, 45-54. https://doi.org/10.7185/genrhe nlet.1705

Bast, R., Scherer, E.E., Sprung, P., Me $\_$rer, K., Fischer-Gödde, M., Taetz, S., Böhnke, M., Schmid-Beurmann, '--., Münker, C., Kleine, T., Srinivasan, G., 2017. Reconciliation of the excess ${ }^{17} \mathrm{j}+\mathrm{t}$ conundrum in meteorites: Recent disturbances of the Lu-Hf and Sm-Nd is itope systematics. Geochim. Cosmochim. Acta 212, 303-323. https://doi.ols/1i.1016/j.gca.2017.05.043

Bischoff, A., Jersek. M , G. au, T., Mirtic, B., Ott, U., Kučera, J., Horstmann, M., Laubenstein, M., r : ’rrmann, S., Řanda, Z., Weber, M., Heusser, G., 2011. Jesenice-A new meteorite fall from Slovenia. Meteorit. Planet. Sci. 46, 793-804. https://doi.org/10.1111/j.1945-5100.2011.01191.x

Bland, P.A., Zolensky, M.E., Benedix, G.K., Sephton, M.A., 2006. Weathering of chondritic meteorites, in: Meteorites and the Early Solar System II. pp. 853-867.

Borg, L.E., Brennecka, G.A., Symes, S.J.K., 2016. Accretion timescale and impact history of Mars deduced from the isotopic systematics of martian meteorites. Geochim. Cosmochim. Acta 175, 150-167. https://doi.org/10.1016/j.gca.2015.12.002

Borg, L.E., Nyquist, L.E., Wiesmann, H., Shih, C.-Y., Reese, Y., 2003. The age of Nd Desert_ Pourkhorsandi et al. 
Dar al Gani 476 and the differentiation history of the martian meteorites inferred from their radiogenic isotopic systematics. Geochim. Cosmochim. Acta 67, 3519-3536. https://doi.org/10.1016/S0016-7037(03)00094-2

Bouvier, A., Gattacceca, J., Agee, C., Grossman, J., Metzler, K., 2017a. The Meteoritical Bulletin, No. 104. Meteorit. Planet. Sci. 52, 2284-2284. https://doi.org/10.1111/maps.12930

Bouvier, A., Gattacceca, J., Grossman, J., Metzler, K., 2017b. The Meteoritical Bulletin, No. 105. Meteorit. Planet. Sci. https://doi.org/10.1111/maps.12944

Bouvier, A., Vervoort, J.D., Patchett, P.J., 2008. The Lu-ht ¿nd Sm-Nd isotopic composition of CHUR: Constraints from unequilibra ?d ', hondrites and implications for the bulk composition of terrestria. bla lets, Earth and Planetary Science Letters. https://doi.org/10.1016/j.eps'..ュふ3.06.010

Brandon, A.D., Nyquist, L.E., Shih, C.-Y., Wiesrıanı, H., 2004. Rb-Sr and Sm-Nd Isotope Systematics of Shergottite NW/, 35 j: Crystallization Age and Implications for Alteration of Hot $\Gamma$ ee: en SNC Meteorites, in: 34th Lunar and

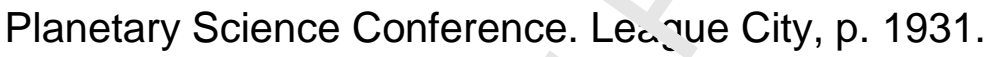

Brandon, A.D., Puchtel, I.S., Wall.e : h.J., Day, J.M.D., Irving, A.J., Taylor, L.A., 2012. Evolution of the ma' "un n Iantle inferred from the 187Re-187Os isotope and highly siderophile $\epsilon$ ment abundance systematics of shergottite meteorites. Geochim. Cosmochim. Auta 76, 206-235. https://doi.org/10. 01i/j.gca.2011.09.047

Cartwright, J. a., Ocker K.D., Crowther, S. a., Burgess, R., Gilmour, J.D., 2010. Terrestrial and Martian weathering signatures of xenon components in shergottite mineral separates. Meteorit. Planet. Sci. 45, 1359-1379. https://doi.org/10.1111/j.1945-5100.2010.01101.x

Chauvel, C., Blichert-Toft, J., 2001. A hafnium isotope and trace element perspective on melting of the depleted mantle. Earth Planet. Sci. Lett. 190, 137-151. https://doi.org/10.1016/S0012-821X(01)00379-X

Crozaz, G., Floss, C., Wadhwa, M., 2003. Chemical alteration and REE mobilization in meteorites from hot and cold deserts. Geochim. Cosmochim. Acta 67, 47274741. https://doi.org/10.1016/j.gca.2003.08.008 
Crozaz, G., Wadhwa, M., 2001. The terrestrial alteration of saharan shergottites dar al ganid 476 and 489: A case study of weathering in a hot desert environment. Geochim. Cosmochim. Acta 65, 971-978. https://doi.org/10.1016/S00167037(00)00586-X

Davis, A.M., Richter, F.M., 2014. Condensation and Evaporation of Solar System Materials, in: Treatise on Geochemistry. Elsevier, pp. 335-360. https://doi.org/10.1016/B978-0-08-095975-7.00112-1

Debaille, V., Brandon, A.D., Yin, Q.Z., Jacobsen, B., 2007. Coupled 142Nd-143Nd evidence for a protracted magma ocean in Mars. Natı e 450, 525-528. https://doi.org/10.1038/nature06317

DePaolo, D.J., 1988. Neodymium Isotope Geochemistry. Minerals and Rocks. Springer Berlin Heidelberg, Berlin, Heidelber;. hi,;s://doi.org/10.1007/978-3642-48916-7

Dreibus, G., Huisl, W., Haubold, R., Jagoutž. ¿.. 2001. Influence of Terrestrial Desert Weathering in Martian Meteorites. $\mathrm{N}$ эt૯:rit. Planet. Sci. 36, A50. https://doi.org/10.1111/j.1945-510u.?001.tb01534.x

Drouard, A., Gattacceca, J., Hutz' ¿, A., Rochette, P., Braucher, R., Bourlès, D., Gounelle, M., Morbidelli, f.., Ttivaille, V., Van Ginneken, M., Valenzuela, M., Quesnel, Y., Martinez, ᄃ., '<ن19. The meteorite flux of the past 2 m.y. recorded in the Atacama Desert Geı.'ogy 47, 673-676. https://doi.org/10.1130/G45831.1

Ebihara, M., and $\mathrm{H}_{\iota} \mathrm{nd}_{i} \quad \mathrm{~N}_{\text {... }}$ 1983. Distribution of rare earth elements and uranium in various component; of ordinary chondrites. Meteoritics 19, 69-77. https://doi.org/10.1111/j.1945-5100.1984.tb00027.x.

Edmunson, J., Borg, L.E., Shearer, C.K., Papike, J.J., 2005. Defining the mechanisms that disturb the $\mathrm{Sm}-\mathrm{Nd}$ isotopic systematics of the Martian meteorites: Examples from Dar al Gani 476 and Allan Hills 77005. Meteorit. Planet. Sci. 40, 1159-1174. https://doi.org/10.1111/j.1945-5100.2005.tb00181.x

Elardo, S.M., Shearer, C.K., Fagan, A.L., Borg, L.E., Gaffney, A.M., Burger, P. V., Neal, C.R., Fernandes, V.A., McCubbin, F.M., 2014. The origin of young mare basalts inferred from lunar meteorites Northwest Africa 4734, 032, and LaPaz Icefield 02205. Meteorit. Planet. Sci. 49, 261-291. 
https://doi.org/10.1111/maps.12239

Folco, L., D'Orazio, M., Perchiazzi, N., 2007. Authenticating the recovery location of meteorites: The case of Castenaso. Meteorit. Planet. Sci. 42, 321-330. https://doi.org/10.1111/j.1945-5100.2007.tb00236.x

Garvie, L.A.J., 2012. The Meteoritical Bulletin, No. 99, April 2012. Meteorit. Planet. Sci. 47, E1-E52. https://doi.org/10.1111/maps.12026

Gattacceca, J., Valenzuela, M., Uehara, M., Jull, A.J.T., Giscard, M., Rochette, P., Braucher, R., Suavet, C., Gounelle, M., Morata, D., Munayco, P., Bourot-Denise, M., Bourles, D., Demory, F., 2011. The densest metevita collection area in hot deserts: The San Juan meteorite field (Atacama De: ert Chile). Meteorit. Planet. Sci. 46, 1276-1287. https://doi.org/10.1111/j.1947-5 00.2011.01229.x

Gibson, E., Bogard, D., 1978. Chemical alteratio 101 the Holbrook chondrite resulting from terrestrial weathering. Meteoritics 13, :77-?89. https://doi.org/10.1111/j.1945-5100.197 .tb J0467.x

Golden, D.C., Ming, D.W., Zolensky, M.F.., 1995. Chemistry and mineralogy of oxidation products on the surface of ine Hoba nickel-iron meteorite. Meteoritics 30, 418-422. https://doi.org/^^ 1 1 1 i1/j.1945-5100.1995.tb01146.x

Gooding, J.L., 1982. Mineralc ical aspects of terrestrial weathering effects in chondrites from Allan Hilli Antarctica, in: Lunar and Planetary Science Conference. pp. 110 s 1:22.

Haloda, J., Týcová, r., hurotev, R.L., Fernandes, V.A., Burgess, R., Thöni, M., Jelenc, M., Jakes, P., Gabzdyl, P., Košler, J., 2009. Petrology, geochemistry, and age of low-Ti mare-basalt meteorite Northeast Africa 003-A: A possible member of the Apollo 15 mare basaltic suite. Geochim. Cosmochim. Acta 73, 3450-3470. https://doi.org/10.1016/j.gca.2009.03.003

Hanson, G.N., 1980. Rare Earth Elements in Petrogenetic Studies of Igneous Systems. Annu. Rev. Earth Planet. Sci. 8, 371-406. https://doi.org/10.1146/annurev.ea.08.050180.002103

Harvey, R., 2003. The Origin and Significance of Antarctic Meteorites. Chemie der Erde - Geochemistry 63, 93-147. 
Hezel, D.C., Schlüter, J., Kallweit, H., Jull, a. J.T., Al Fakeer, O.Y., Al Shamsi, M., Strekopytov, S., 2011. Meteorites from the United Arab Emirates: Description, weathering, and terrestrial ages. Meteorit. Planet. Sci. 46, 327-336. https://doi.org/10.1111/j.1945-5100.2010.01165.x

Hofmann, B.A., Gnos, E., Jull, A.J.T., Szidat, S., Majoub, A., Al Wagdani, K., Habibullah, S.N., Halawani, M., Hakeem, M., Al Shanti, M., Al Solami, A., 2018. Meteorite reconnaissance in Saudi Arabia. Meteorit. Planet. Sci. 53, 2372-2394. https://doi.org/10.1111/maps.13132

Hutzler, A., Gattacceca, J., Rochette, P., Braucher, R., Cà o, B., Christensen, E.J., Cournede, C., Gounelle, M., Laridhi Ouazaa, N., Mi. rtır ?z, R., Valenzuela, M., Warner, M., Bourles, D., 2016. Description of a very dense meteorite collection area in western Atacama: Insight into the long-i rm composition of the meteorite flux to Earth. Meteorit. Planet. Sci. 51, 468- '82.

https://doi.org/10.1111/maps.12607

Jagoutz, E., Jotter, R., Dreibus, G., Zartm १, K., 2001. New U-PB Isotope data on SNC meteorites. 32nd Annu. Luı,r Planet. Sci. Conf. A1307.

Jochum, K.P., Weis, U., Schwager L. Stoll, B., Wilson, S.A., Haug, G.H., Andreae, M.O., Enzweiler, J., 2016. Ref ar snce Values Following ISO Guidelines for Frequently Requested Po K Reference Materials. Geostand. Geoanalytical Res. 40, 333-350. https://du : 01 y/10.1111/j.1751-908X.2015.00392.x

Jones, R.H., McCubbiı, F. Y., Dreeland, L., Guan, Y., Burger, P.V., Shearer, C.K., 2014. Phosphate 1 . inerals in LL chondrites: A record of the action of fluids during metamorphism on ordinary chondrite parent bodies. Geochim. Cosmochim. Acta 132, 120-140. https://doi.org/10.1016/j.gca.2014.01.027

Jull, A. J.T., Giscard, M.D., Hutzler, A., Schnitzer, C.J., Zahn, D., Burr, G.S., Mchargue, L.R., Hill, D., 2013. Radionuclide studies of stony meteorites from hot deserts. pp. 1779-1789. https://doi.org/10.2458/azu_js_rc.55.16202

Kagi, H., Takahashi, K., 1998. Relationship between positive cerium anomaly and adsorbed water in Antarctic lunar meteorites. Meteorit. Planet. Sci. 33, 10331040. https://doi.org/10.1111/j.1945-5100.1998.tb01710.x

Kaneoka, I., 1983. Anomalously old 40Ar-39Ar ages of Antarctic meteorites due to 
weathering. Nature 304, 146-148. https://doi.org/10.1038/304146a0

King, A.J., Phillips, K.J.H., Strekopytov, S., Vita-Finzi, C., Russell, S.S., 2020.

Terrestrial modification of the Ivuna meteorite and a reassessment of the chemical composition of the $\mathrm{Cl}$ type specimen. Geochim. Cosmochim. Acta 268, 73-89. https://doi.org/10.1016/j.gca.2019.09.041

Koeberl, C., Cassidy, W.A., 1991. Proceedings of a workshop on Differences Between Antarctic and Non-Antarctic Meteorites 55, 3-18. https://doi.org/10.1016/0016-7037(91)90395-L

Kuznetsov, R., 2011. Cosmochemistry of thorium, samariurı, and cesium in the substance of ordinary chondrites and problem of dis, nos al of actinide and fission product concentrates in the Earth crust. Radioch 、mis try 53, 81-86. https://doi.org/10.1134/S1066362211010115

Martin, C., Debaille, V., Lanari, P., Goderis, S., ' 'anc'endael, I., Vanhaecke, F., Vidal, O., Claeys, P., 2013. Geochim. Cosm x'.ır. Acta 120, 496-513. https://doi.org/10.1016/j.gca.201?.vi.0us

Mittlefehldt, D.W., Lindstrom, M.M. 1991. Generation of abnormal trace element abundances in Antarctic euc'it s by weathering processes. Geochim. Cosmochim. Acta 55, 77-ð_ hiips://doi.org/10.1016/0016-7037(91)90401-P

Moore, C.B., Brown, H., 1950. Barium in stony meteorites. J. Geophys. Res. 68, 4293-4296. https://dui nrg/10.1029/JZ068i014p04293

Munayco, P., Munayci u., de Avillez, R.R., Valenzuela, M., Rochette, P., Gattacceca, J., Surzelli, R.B., 2013. Weathering of ordinary chondrites from the Atacama Desert, Chile, by Mössbauer spectroscopy and synchrotron radiation X-ray diffraction. Meteorit. Planet. Sci. 48, 457-473.

https://doi.org/10.1111/maps.12067

Patchett, P.., Vervoort, J.., Söderlund, U., Salters, V.J.., 2004. Lu-Hf and Sm-Nd isotopic systematics in chondrites and their constraints on the $\mathrm{Lu}-\mathrm{Hf}$ properties of the Earth. Earth Planet. Sci. Lett. 222, 29-41. https://doi.org/10.1016/j.epsl.2004.02.030

Pourkhorsandi, H., D’Orazio, M., Rochette, P., Valenzuela, M., Gattacceca, J., 
Mirnejad, H., Sutter, B., Hutzler, A., Aboulahris, M., 2017a. Modification of REE distribution of ordinary chondrites from Atacama (Chile) and Lut (Iran) hot deserts: Insights into the chemical weathering of meteorites. Meteorit. Planet. Sci. 52, 1843-1858. https://doi.org/10.1111/maps.12894

Pourkhorsandi, H., Gattacceca, J., Devouard, B., D'Orazio, M., Rochette, P., Beck, P., Sonzogni, C., Valenzuela, M., 2017b. The ungrouped chondrite El Médano 301 and its comparison with other reduced ordinary chondrites. Geochim. Cosmochim. Acta 218, 98-113. https://doi.org/10.1016/j.gca.2017.09.013

Pourkhorsandi, H., Gattacceca, J., Rochette, P., D'Orazio, V., Kamali, H., Avillez, R., Letichevsky, S., Djamali, M., Mirnejad, H., Debaille, V., Iull, A.J.T., 2019. Meteorites from the Lut Desert (Iran). Meteorit. Piarıt. Sci. maps.13311. https://doi.org/10.1111/maps.13311

Ruzicka, A., Grossman, J., Bouvier, A., Agee, C.t. 2017. The Meteoritical Bulletin, No. 103. Meteorit. Planet. Sci. 52, 1014-7 J14. https://doi.org/10.1111/maps.1288尺

Ruzicka, A., Grossman, J., Bouvier, A., :'terd, C.D.K., Agee, C.B., 2015a. The Meteoritical Bulletin, No. 101. Nc +eorit. Planet. Sci. 50, 1661-1661. https://doi.org/10.1111/mans. ${ }^{12}, 90$

Ruzicka, A., Grossman, J., E-ouviar, A., Herd, C.D.K., Agee, C.B., 2015b. The Meteoritical Bulletin, No. 102. Meteorit. Planet. Sci. 50, 1662-1662. https://doi.org/10. $11: /$ maps.12491

Ruzicka, A., Grossman J.N., Garvie, L., 2014. The Meteoritical Bulletin, No. 100, 2014 June. Meteorit. Planet. Sci. 49, E1-E101. https://doi.org/10.1111/maps.12342

Saunier, G., Poitrasson, F., Moine, B., Gregoire, M., Seddiki, A., 2010. Effect of hot desert weathering on the bulk-rock iron isotope composition of $\mathrm{L} 6$ and $\mathrm{H} 5$ ordinary chondrites. Meteorit. Planet. Sci. 45, 195-209. https://doi.org/10.1111/j.1945-5100.2010.01017.x

Schultz, L., Franke, L., Bevan, A.W.R., 2005. Noble gases in ten Nullarbor chondrites: Exposure ages, terrestrial ages, and weathering effects. Meteorit. Planet. Sci. 40, 659-664. https://doi.org/10.1111/j.1945-5100.2005.tb00971.x 
Shih, C.-Y., Nyquist, L.E., Reese, Y., 2007. Rb-Sr and Sm-Nd Isotopic Studies of Martian Depleted Shergottites SaU 094/005, in: 38th Lunar and Planetary Science Conference. League City, p. 1745.

Shimizu, H., Masuda, A., Tanaka, T., 1983. Cerium anomaly in REE pattern of Antarctic eucrite, in: 8th Symposium on Antarctic Meteorites, National Institute of Polar Research. pp. 341-348.

Sokol, A.K., Fernandes, V.A., Schulz, T., Bischoff, A., Burgess, R., Clayton, R.N., Münker, C., Nishiizumi, K., Palme, H., Schultz, L., Weckwerth, G., Mezger, K., Horstmann, M., 2008. Geochemistry, petrology and ár, $ə s$ of the lunar meteorites Kalahari 008 and 009: New constraints on early lun ir $\in$ 'olution. Geochim. Cosmochim. Acta 72, 4845-4873. https://doi.org IU.1016/j.gca.2008.07.012

Stelzner, T., Heide, K., 1996. The study of weathr.:iny products of meteorites by means of evolved gas analysis. Meteorit. Pla1 $-i$. Sci. 31, 249-254. https://doi.org/10.1111/j.1945-5100.198 o..202020.x

Stelzner, T., Heide, K., Bischoff, A., W'ex ?r, D., Scherer, P., Schultz, L., Happel, M., Schron, W., Neupert, U., Michel, K., Clayton, R.N., Mayeda, T.K., Bonani, G., Haidas, I., Ivy-Ochs, S., Suter N: 1999. An interdisciplinary study of weathering effects in ordinary chondrites $f \circ \mathrm{n}$ the Acfer region, Algeria. Meteorit. Planet. Sci. 34, 787-794. https:// . ר. org/10.1111/j.1945-5100.1999.tb01391.x

Stephant, A., Garvie, L.A J., । 'ane, P., Hervig, R., Wadhwa, M., 2018. Terrestrial exposure of a fres $1 N_{1}$ rrtian meteorite causes rapid changes in hydrogen isotopes and wate. concentrations. Sci. Rep. 8, 12385.

https://doi.org/10.1038/s41598-018-30807-w

Tatsumoto, M., Unruh, D.M., Patchett, P.J., 1981. U-Pb and Lu-Hf systematics of Antarctic meteorites. Mem. Natl. Inst. Polar Res. Spec. issue 20, 237-249.

Torigoye-Kita, N., Misawa, K., Tatsumoto, M., 1995. U-Th-Pb and Sm-Nd isotopic systematics of the Goalpara ureilite: Resolution of terrestrial contamination. Geochim. Cosmochim. Acta 59, 381-390. https://doi.org/10.1016/00167037(95)00279-9

Velbel, M. a., Long, D.T., Gooding, J.L., 1991. Terrestrial weathering of Antarctic stone meteorites - Formation of Mg-carbonates on ordinary chondrites. 
Geochim. Cosmochim. Acta 55, 67-76. https://doi.org/10.1016/00167037(91)90400-Y

Velbel, M. a, 2014. Terrestrial weathering of ordinary chondrites in nature and continuing during laboratory storage and processing: Review and implications for Hayabusa sample integrity. Meteorit. Planet. Sci. 49, 154-171. https://doi.org/10.1111/j.1945-5100.2012.01405.x

Wainwright, A.N., El Atrassi, F., Debaille, V., Mattielli, N., 2019. Geochemistry and petrogenesis of Archean mafic rocks from the Amsaga area, West African craton, Mauritania. Precambrian Res. 324, 208-219. https://doi.org/10.1016/j.precamres.2019.02.005

Walker, R.J., Yin, Q.-Z., Heck, P.R., 2018. Rapid effe ts ' ff terrestrial alteration on highly siderophile elements in the Sutter's Mi!. $\eta_{c}{ }^{+}$eorite. Meteorit. Planet. Sci. 53, 1500-1506. https://doi.org/10.1111/maps. ${ }^{+}$s102

Wasson, J.T., Kallemeyn, G.W., 1988. Comp'ssi ions of Chondrites. Philos. Trans. R. Soc. A Math. Phys. Eng. Sci. 325, 5. $5-544$. https://doi.org/10.1098/rsta.1988.0 $\uparrow 6$

Weber, P., Hofmann, B.A., Tolba I. Vuilleumier, J.-L., 2017. A gamma-ray spectroscopy survey of Or،anl ıneteorites. Meteorit. Planet. Sci. 52, 1017-1029. https://doi.org/10.1111/: /aps.12847

Weisberg, M.K., McCoy. I.I Krot, A.N., 2006. Systematics and evaluation of meteorite class 'fic.tio' I, in: Lauretta, D.S., McSween, Jr., H.Y. (Eds.), Meteorites and the Early Solar System II. University of Arizona Press, Tucson, pp. 19-52.

Welten, K.C., Alderliesten, C., Borg, K. Van Der, Lindner, L., Loeken, T., Schultz, L., 1997. Lewis Cliff 86360: An Antarctic L-chondrite with a terrestrial age of 2.35 million years. Meteorit. Planet. Sci. 32, 775-780.

Wilson, S.A., 1997a. Data compilation for USGS reference material BHVO-2, Hawaiian Basalt, in: US Geological Survey Open-File Report.

Wilson, S.A., 1997b. The collection, preparation, and testing of USGS reference material BCR-2, Columbia River Basalt, in: US Geological Survey Open-File Report. 
Wlotzka, F., 1993. A weathering scale for the ordinary chondrites. Meteoritics.

Zekollari, H., Goderis, S., Debaille, V., van Ginneken, M., Gattacceca, J., ASTER team, Jull, A.J.T., Lenaerts, J.T.M., Yamaguchi, A., Claeys, P., 2019. Unravelling the high-altitude Nansen blue ice field meteorite trap (East Antarctica) and implications for regional palaeo-conditions. Earth Planet. Sci. Lett. 248, 289-310. https://doi.org/10.1016/j.gca.2018.12.035

Zeng, X., Li, S., Leya, I., Wang, S., Smith, T., Li, Y., Wang, P., 2018. The Kumtag 016 L5 strewn field, Xinjiang Province, China. Meteorit. Planet. Sci. 53, 11131130. https://doi.org/10.1111/maps.13073

Zurfluh, F.J., Hofmann, B. a., Gnos, E., Eggenberger, U., 20 11. Evaluation of the utility of handheld XRF in meteoritics. X-Ray Spe ?tro n. 40, 449-463. https://doi.org/10.1002/xrs.1369

Zurfluh, F.J., Hofmann, B.A., Gnos, E., Eggenberge, U., Greber, N.D., Villa, I.M., 2012. Weathering and Strontium Contar, $r$ : tion of Meteorites Recovered in the Sultanate of Oman. Meteorite 20r,v, 34- 38. 